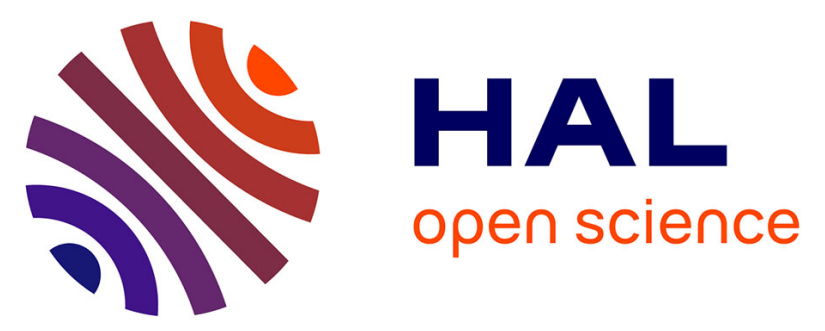

\title{
Itô SDE-based generator for a class of non-Gaussian vector-valued random fields in uncertainty quantification
}

J. Guilleminot, Christian Soize

\section{To cite this version:}

J. Guilleminot, Christian Soize. Itô SDE-based generator for a class of non-Gaussian vector-valued random fields in uncertainty quantification. SIAM Journal on Scientific Computing, 2014, Methods and Algorithms for Scientific Computing, 36 (6), pp.A2763-A2786. 10.1137/130948586 . hal-01100173

\section{HAL Id: hal-01100173 \\ https://hal.science/hal-01100173}

Submitted on 6 Jan 2015

HAL is a multi-disciplinary open access archive for the deposit and dissemination of scientific research documents, whether they are published or not. The documents may come from teaching and research institutions in France or abroad, or from public or private research centers.
L'archive ouverte pluridisciplinaire HAL, est destinée au dépôt et à la diffusion de documents scientifiques de niveau recherche, publiés ou non, émanant des établissements d'enseignement et de recherche français ou étrangers, des laboratoires publics ou privés. 


\title{
ITÔ SDE-BASED GENERATOR FOR A CLASS OF NON-GAUSSIAN VECTOR-VALUED RANDOM FIELDS IN UNCERTAINTY QUANTIFICATION*†
}

\author{
JOHANN GUILLEMINOT AND CHRISTIAN SOIZE
}

\begin{abstract}
This paper is concerned with the derivation of a generic sampling technique for a class of non-Gaussian vector-valued random fields. Such an issue typically arises in uncertainty quantification for complex systems, where the input coefficients associated with the elliptic operators must be identified by solving statistical inverse problems. Specifically, we consider the case of non-Gaussian random fields with values in some arbitrary bounded or semi-bounded subsets of $\mathbb{R}^{n}$. The approach involves two main features. The first one is the construction of a family of random fields converging, at a user-controlled rate, towards the target random field. Each of these auxialiary random fields can be subsequently simulated by solving a family of Itô stochastic differential equations. The second ingredient is the definition of an adaptive discretization algorithm. The latter allows refining the integration step on-the-fly and prevents the scheme from diverging. The proposed strategy is finally exemplified on three examples, each of which serving as a benchmark, either for the adaptivity procedure or for the convergence of the diffusions.
\end{abstract}

Key words. random field, Itô stochastic differential equation, adaptive algorithm, uncertainty quantification

\section{Introduction.}

1.1. Notation. For later reference, the following matrix sets are introduced:

(i) $\mathbb{M}_{q}(\mathbb{R})$ is the set of all the $(q \times q)$ real matrices.

(ii) $\mathbb{M}_{q}^{S}(\mathbb{R})$ is the set of all the $(q \times q)$ symmetric real matrices.

(iii) $\mathbb{M}_{q}^{+}(\mathbb{R})$ is the set of all the $(q \times q)$ symmetric positive-definite real matrices. For $\boldsymbol{x}$ and $\boldsymbol{y}$ in $\mathbb{R}^{n}$, we denote by $\langle\boldsymbol{x}, \boldsymbol{y}\rangle=\sum_{i=1}^{n} x_{i} y_{i}$ and $\|\boldsymbol{x}\|=\langle\boldsymbol{x}, \boldsymbol{x}\rangle^{1 / 2}$ the Euclidean inner product and the associated norm, respectively. Let $\left\{\boldsymbol{e}^{1}, \ldots, \boldsymbol{e}^{n}\right\}$ be the canonical basis of $\mathbb{R}^{n}$. The null and identity $(q \times q)$ matrices are denoted by $\left[0_{q}\right]$ and $\left[I_{q}\right]$. Let $a \wedge b=\min (a, b)$ and $a \vee b=\max (a, b)$. Notations $c, c_{\boldsymbol{x}}$ and $c_{\boldsymbol{x}}^{\epsilon}$ are used to denote normalization constants, the values of which may vary from line to line. Let $\mathbb{E}$ denote the mathematical expectation.

Let $\left\{\boldsymbol{\Xi}(\mathbf{x}), \boldsymbol{x} \in \mathbb{R}^{d}\right\}, d \geqslant 1$, be a second-order mean-square continuous centered homogeneous $\mathbb{R}^{n}$-valued Gaussian random field, defined on a probability space $(\Theta, \mathcal{T}, \mathcal{P})$ by a continuous $\mathbb{M}_{n}(\mathbb{R})$-valued normalized correlation function $\left(\boldsymbol{x}, \boldsymbol{x}^{\prime}\right) \mapsto$ $\left[R_{\Xi}\left(\boldsymbol{x}-\boldsymbol{x}^{\prime}\right)\right]$ such that

$$
\left[R_{\Xi}\left(\boldsymbol{x}-\boldsymbol{x}^{\prime}\right)\right]=\operatorname{diag}\left(\mathfrak{R}_{1}\left(\boldsymbol{x}-\boldsymbol{x}^{\prime}\right), \ldots, \mathfrak{R}_{n}\left(\boldsymbol{x}-\boldsymbol{x}^{\prime}\right)\right), \quad \forall\left(\boldsymbol{x}, \boldsymbol{x}^{\prime}\right) \in \mathbb{R}^{d} \times \mathbb{R}^{d}
$$

wherein each normalized correlation function $\boldsymbol{x} \mapsto \mathfrak{R}_{i}(\boldsymbol{x})$ is defined from $\mathbb{R}^{d}$ into $[-1,1]$ and is such that $\mathfrak{R}_{i}(\mathbf{0})=1$ for $1 \leqslant i \leqslant n$ (hence, the components of $\{\boldsymbol{\Xi}(\mathbf{x}), \boldsymbol{x} \in$ $\left.\mathbb{R}^{d}\right\}$ are mutually independent Gaussian random fields). We denote by $\boldsymbol{x} \mapsto \boldsymbol{\Xi}\left(\boldsymbol{x}, \theta_{r}\right)$ the $r$-th independent realization of this Gaussian random field. Accordingly, and for any fixed value of $\boldsymbol{x}, \boldsymbol{\Xi}_{\boldsymbol{x}}\left(\theta_{r}\right)$ denotes the $r$-th realization of the $\mathbb{R}^{n}$-valued Gaussian random variable $\boldsymbol{\Xi}_{\boldsymbol{x}}=\boldsymbol{\Xi}(\boldsymbol{x})$.

*PREPRINT VERSION (SEE SIAM J. SCI. COMPUT. 2014 SOCIETY FOR INDUSTRIAL AND APPLIED MATHEMATICS VOL. 36, NO. 6, PP. A2763-A2786).

${ }^{\dagger}$ This work was supported by the French National Research Agency (ANR) under TYCHE and MOSAIC contracts.

${ }^{\ddagger}$ Université Paris-Est, Laboratoire Modélisation et Simulation Multi Echelle, MSME UMR 8208 CNRS, 5 Bd Descartes, 77454 Marne la Vallée, France. 
1.2. Preliminaries. This work addresses the random generation of a large class of random fields with values in some subset of $\mathbb{R}^{n}$. Specifically, it is concerned with the simulation of a class of random fields:

- That are non-Gaussian and of second-order.

- For which the family of first-order marginal probability distributions (m.p.d.f.) and spatial correlation lengths must be prescribed.

- That contains the particular case of homogeneous random fields (for the translation in $\mathbb{R}^{d}$ ).

Models belonging to such a class are referred to as Prior Algebraic Stochastic Models (PASMs) in the literature of uncertainty quantification (UQ) and are typically devoted to identification solving underdetermined statistical inverse problems [47] [40]. In order to fix the main ideas and concepts that will be discussed throughout, we first denote by $\{\boldsymbol{A}(\boldsymbol{x}), \boldsymbol{x} \in \Omega\}$ the non-Gaussian random field, defined on $(\Theta, \mathcal{T}, \mathcal{P})$, to be generated. It is assumed that this random field takes its values in some set $\mathcal{S} \in \mathbb{R}^{n}$ and is indexed by an open bounded domain $\Omega$ in $\mathbb{R}^{d}$. Let $\mathcal{S}^{\circ}, \overline{\mathcal{S}}$ and $\partial \mathcal{S}=\overline{\mathcal{S}} \backslash \mathcal{S}^{\circ}$ denote the interior, closure and boundary of $\mathcal{S}$, respectively. The notation $\boldsymbol{x} \downarrow \partial \mathcal{S}$ means that $\boldsymbol{x}$ approaches the boundary $\partial \mathcal{S}$ from $\mathcal{S}^{\circ}$. Let $\left\{p^{\mathrm{t}}(\cdot ; \boldsymbol{x})\right\}_{\boldsymbol{x} \in \Omega}$ be the prescribed family of first-order m.p.d.f. For most UQ applications, the aforementionned random field corresponds to a vector-valued representation of a random field with values in some set $\widetilde{\mathbb{M}}_{q}^{+}(\mathbb{R}) \subseteq \mathbb{M}_{q}^{+}(\mathbb{R})$ (with $n \leqslant q(q+1) / 2$ ), the matrix-valued random field being typically interpreted as the stochastic coefficient of an elliptic operator.

As a matter of illustration, let $\{[\boldsymbol{A}(\boldsymbol{x})], \boldsymbol{x} \in \Omega\}$ be a random field with values in $\widetilde{\mathbb{M}}_{3}^{+}(\mathbb{R}) \subset \mathbb{M}_{3}^{+}(\mathbb{R})$ such that:

$$
[\boldsymbol{A}(\boldsymbol{x})]=\left[\begin{array}{ccc}
A_{1}(\boldsymbol{x}) & A_{2}(\boldsymbol{x}) & 0 \\
A_{2}(\boldsymbol{x}) & A_{3}(\boldsymbol{x}) & 0 \\
0 & 0 & A_{4}(\boldsymbol{x})
\end{array}\right], \quad \forall \boldsymbol{x} \in \Omega .
$$

Let $\boldsymbol{a}=\left(a_{1}, a_{2}, a_{3}, a_{4}\right) \in \mathbb{R}^{4}$. The random field $\{\boldsymbol{A}(\boldsymbol{x}), \boldsymbol{x} \in \Omega\}$ such that $\boldsymbol{A}(\boldsymbol{x})=$ $\left(A_{1}(\boldsymbol{x}), A_{2}(\boldsymbol{x}), A_{3}(\boldsymbol{x}), A_{4}(\boldsymbol{x})\right)$ for all $\boldsymbol{x}$ in $\Omega$ thus takes its values in

$$
\mathcal{S}=\left\{\boldsymbol{a} \in \mathbb{R}^{4} \mid a_{1}>0, a_{3}>0, a_{1} a_{3}-a_{2}^{2}>0, a_{4}>0\right\},
$$

and

$$
\overline{\mathcal{S}}=\left\{\boldsymbol{a} \in \mathbb{R}^{4} \mid a_{1} \geqslant 0, a_{3} \geqslant 0, a_{1} a_{3}-a_{2}^{2} \geqslant 0, a_{4} \geqslant 0\right\} .
$$

This basic example shows that even for a simple case, the boundary $\partial \mathcal{S}$ can be rather complex, and it is worth mentioning that this complexity can fairly be expected to grow significantly as the dimension $n$ increases.

1.3. Overview of the approach. The generation of non-Gaussian stochastic processes or random fields has been extensively discussed in the literature, at least for the case $\mathcal{S}=\mathbb{R}^{n}$. A first class of algorithms is based on the construction of nonlinear memoryless transformations acting on Gaussian models (see [3] [42] for a recent survey, among others). Such transformations can be defined, for instance, through Hermite series expansions (see [18] [22] [41] and the references therein). The parameters of the underlying Gaussian model(s), as well as the coefficients of the expansions, are then numerically calibrated so that the image by the nonlinear mapping exhibits some target probabilistic properties - in most cases, a target marginal distribution and a specified correlation structure. In the present case where only the system of first-order 
marginal distributions is concerned, and since $\boldsymbol{A}(\boldsymbol{x})$ is a second-order vector-valued random variable, a convenient way to define such a mapping is to have recourse to a pointwise polynomial Chaos expansion (PCE) [20] (see also [46] and [32]):

$$
\boldsymbol{A}(\boldsymbol{x})=\sum_{\boldsymbol{\alpha},|\boldsymbol{\alpha}|=0}^{+\infty} \boldsymbol{a}_{\boldsymbol{\alpha}}(\boldsymbol{x}) h_{\boldsymbol{\alpha}}\left(\boldsymbol{\Xi}_{\boldsymbol{x}}\right), \quad \forall \boldsymbol{x} \in \Omega
$$

in which $\boldsymbol{\alpha}=\left(\alpha_{1}, \ldots, \alpha_{n}\right) \in \mathbb{N}^{n}$ is a multi-index with modulus $|\boldsymbol{\alpha}|=\sum_{i} \alpha_{i}$ and $\left\{h_{\boldsymbol{\alpha}}\right\}_{\boldsymbol{\alpha}}$ are the multidimensional Hermite polynomials. In effect, the mean-square convergent expansion defined by Eq. (1.5) is truncated after polynomials of degree $N_{p c e}$, that is:

$$
\boldsymbol{A}(\boldsymbol{x}) \simeq \sum_{\boldsymbol{\alpha},|\boldsymbol{\alpha}|=0}^{N_{p c e}} \boldsymbol{a}_{\boldsymbol{\alpha}}(\boldsymbol{x}) h_{\boldsymbol{\alpha}}\left(\boldsymbol{\Xi}_{\boldsymbol{x}}\right), \quad \forall \boldsymbol{x} \in \Omega
$$

From a random generation point of view, the use of such an expansion is certainly very efficient, as it can easily be implemented using fast matrix-based operations. In addition, the generation of a realization of $\{\boldsymbol{A}(\boldsymbol{x}), \boldsymbol{x} \in \Omega\}$ requires the simulation of a single realization of the Gaussian random field $\left\{\boldsymbol{\Xi}(\mathbf{x}), \boldsymbol{x} \in \mathbb{R}^{d}\right\}$ only - as opposed to the second class of algorithms detailed below. However, one should note that the use of PCE rises some practical numerical difficulties which may degrade the sampling quality, among which the identification of the deterministic fields $\left\{\boldsymbol{x} \mapsto \boldsymbol{a}_{\alpha}(\boldsymbol{x})\right\}_{\alpha}$ (even for moderate values of $n$ ) and numerical convergence issues to name a few.

A second general class of techniques involves transformations with memory and essentially relies on the construction of stochastic differential equations. The algorithm which is proposed and numerically studied in this paper belongs to this second class of numerical strategies and is devoted to the case where $\mathcal{S}$ is any bounded or semi-bounded part of $\mathbb{R}^{n}$. Broadly speaking, the approach consists in prescribing the family of target first-order marginal probability distributions as the family of invariant measures associated with a family of diffusion processes, the latter being defined as the stationary solutions of a family of Itô stochastic differential equations (ISDEs). Conceptually, such an approach amounts to alternatively define the random field $\{\boldsymbol{A}(\boldsymbol{x}), \boldsymbol{x} \in \Omega\}$ through the following mapping:

$$
\boldsymbol{A}(\boldsymbol{x})=\mathcal{H}_{\boldsymbol{x}}(\{\boldsymbol{W}(r, \boldsymbol{x}), r \geq 0\}), \quad \forall \boldsymbol{x} \in \Omega,
$$

with $\mathcal{H}_{\boldsymbol{x}}$ a nonlinear operator and $\{\boldsymbol{W}(r, \boldsymbol{x}), r \geq 0\}$ a $\mathbb{R}^{n}$-valued normalized Wiener process defined on $(\Theta, \mathcal{T}, \mathcal{P})$. The family $\{\{\boldsymbol{W}(r, \boldsymbol{x}), r \geq 0\}\}_{\boldsymbol{x} \in \Omega}$ of Wiener processes thus introduced will be defined more precisely in $\S 2.1$.

1.4. Outline. The outline of the paper, along with the intended contributions of this work, are as follows:

- In $\S 2$, the theoretical background used for constructing each ISDE in the so-called free case (that is, for $\mathcal{S}=\mathbb{R}^{n}$ ) is first recalled for completeness. Specifically, we propose a particular definition of a family of Wiener processes that allows to generate spatial dependendies while solving the family of ISDEs. The definition of the latter is further addressed, and general assumptions related to the existence and uniqueness of invariant measures are recalled. 
- Section 3 is concerned with the constrainted situation where $\mathcal{S}$ is any part of $\mathbb{R}^{n}$. In order to handle this case, an approximating family of non-Gaussian random fields, the definition of which is provided in $\S 3.2$, is introduced. The strategy involves the construction of a family of approximating potential functions through a regularization procedure defined in $\S 3.3$.

- In $\S 4$, we propose a novel adaptive integration algorithm for the family of ISDEs. The adaptation is performed on-the-fly, depending on the current values of the drift coefficients. It prevents from using a too small integration step - while ensuring a reasonably fast convergence towards the stationary solutions.

- The proposed numerical strategy is finally exemplified through three applications in $\S 5$. The first one deals with the restriction of a Gaussian bivariate random variable to a compact support. The latter, as well as the imposed variances, are calibrated such that the particle reaches the boundary of admissible domain $\mathcal{S}$ with a quite large probability, hence providing a benchmark for the regularization and adaptivity procedures. The second application addresses a regularization on a matrix manifold. In particular, a reference generator is used to illustrate the convergence in probability distribution for the proposed algorithms. The last exemple exemplifies the simulation of a non-Gaussian random field with values in the positive-definite cone.

\section{Theoretical background for the free case.}

2.1. Definition of a family of Wiener processes. Let $\boldsymbol{W}=\{\boldsymbol{W}(r, \boldsymbol{x})=$ $\left.\left(W_{1}(r, \boldsymbol{x}), \ldots, W_{n}(r, \boldsymbol{x})\right), r \in \mathbb{R}^{+}, \boldsymbol{x} \in \Omega\right\}$ be a $\mathbb{R}^{n}$-valued centered second-order Gaussian random field such that:

(i) For all $\boldsymbol{x}$ in $\Omega, \boldsymbol{W}(0, \boldsymbol{x})=\mathbf{0}$ a.s.

(ii) The generalized $r$-derivative $\mathrm{D}_{r} \boldsymbol{W}$ of $\boldsymbol{W}$ is the cylindrical normalized Gaussian white noise $\boldsymbol{N}$ (see e.g. [8]).

The covariance generalized function $\left[\mathrm{C}_{\boldsymbol{N}}\right]$ of $\boldsymbol{N}$ is written as

$$
\forall\left(\boldsymbol{x}, \boldsymbol{x}^{\prime}\right) \in \Omega \times \Omega, \quad \forall \tau \in \mathbb{R}, \quad\left[\mathrm{C}_{\boldsymbol{N}}\left(\boldsymbol{x}, \boldsymbol{x}^{\prime}, t+\tau, t\right)\right]=\delta_{0}(\tau)\left[\mathrm{R}_{\boldsymbol{N}}\left(\boldsymbol{x}, \boldsymbol{x}^{\prime}\right)\right],
$$

where $\delta_{0}$ is the Dirac generalized function at the origin of $\mathbb{R}$. In Eq. (2.1), [ $\left.\mathrm{R}_{\boldsymbol{N}}\right]$ denotes the continuous $\mathbb{M}_{n}(\mathbb{R})$-valued function defined on $\Omega \times \Omega$ as

$$
\left[\mathrm{R}_{N}\left(\boldsymbol{x}, \boldsymbol{x}^{\prime}\right)\right]=\left[R_{\Xi}\left(\boldsymbol{x}-\boldsymbol{x}^{\prime}\right)\right],
$$

where $\left[R_{\boldsymbol{\Xi}}\right]$ is the correlation function of the homogeneous Gaussian random field $\left\{\boldsymbol{\Xi}(\boldsymbol{x}), \boldsymbol{x} \in \mathbb{R}^{d}\right\}$ defined in $\S 1.2$. It follows that for all $\boldsymbol{x}$ fixed in $\Omega,\{\boldsymbol{W}(r, \boldsymbol{x}), r \geq 0\}$ is a normalized $\mathbb{R}^{n}$-valued Wiener process, that is, $\{\boldsymbol{W}(r, \boldsymbol{x}), r \geq 0\}$ satisfies the following properties:

(i) The $n$ real-valued stochastic processes $\left\{W_{1}(r, \boldsymbol{x}), r \geqslant 0\right\}, \ldots,\left\{W_{n}(r, \boldsymbol{x}), r \geqslant\right.$ $0\}$ are mutually independent.

(ii) $\boldsymbol{W}(0, \boldsymbol{x})=\mathbf{0}$ a.s.

(iii) The process $\{\boldsymbol{W}(r, \boldsymbol{x}), r \geqslant 0\}$ has independent increments.

(iv) For all $0 \leqslant s<t<+\infty$, the increment $\Delta \boldsymbol{W}(s, t, \boldsymbol{x})=\boldsymbol{W}(t, \boldsymbol{x})-\boldsymbol{W}(s, \boldsymbol{x})$ is a $\mathbb{R}^{n}$-valued second-order random variable which is Gaussian, centered and whose covariance matrix reads as $(t-s)\left[I_{n}\right]$.

Note that $\left\{\boldsymbol{W}(r, \boldsymbol{x}), \boldsymbol{x} \in \mathbb{R}^{d}\right\}$ is then a homogeneous colored Gaussian stochastic field for any fixed value of $r \in \mathbb{R}^{+}$. 
2.2. Construction of a family of ISDEs. Throughout this section, we set $\mathcal{S}=\mathbb{R}^{n}$. It is assumed that for all $\boldsymbol{x}$ in $\Omega$, each element $p^{\mathrm{t}}(\cdot ; \boldsymbol{x})$ of the family of prescribed m.p.d.f. takes the following algebraic form:

$$
p^{\mathrm{t}}(\boldsymbol{u} ; \boldsymbol{x})=c_{\boldsymbol{x}} \exp \{-\Phi(\boldsymbol{u} ; \boldsymbol{x})\}, \quad \forall \boldsymbol{u} \in \mathbb{R}^{n},
$$

in which $\{\Phi(\cdot ; \boldsymbol{x})\}_{\boldsymbol{x} \in \Omega}$ is a family of continuous real-valued functions defined on $\mathbb{R}^{n}$. In what follows, those functions are classically referred to as the potential functions.

Let $\mathcal{F}^{\mathrm{ISDE}}$ be a family of Itô stochastic differential equations indexed by $\boldsymbol{x} \in \Omega$, each element of which writes for $r>0$ :

$$
\begin{aligned}
\mathrm{d} \boldsymbol{U}(r, \boldsymbol{x}) & =\boldsymbol{V}(r, \boldsymbol{x}) \mathrm{d} r, \\
\mathrm{~d} \boldsymbol{V}(r, \boldsymbol{x}) & =\left(-\nabla_{\boldsymbol{u}} \Phi(\boldsymbol{U}(r, \boldsymbol{x}) ; \boldsymbol{x})-\frac{f_{\boldsymbol{x}}}{2} \boldsymbol{V}(r, \boldsymbol{x})\right) \mathrm{d} r+\sqrt{f_{\boldsymbol{x}}} \mathrm{d} \boldsymbol{W}(r, \boldsymbol{x}),
\end{aligned}
$$

where $\left\{f_{\boldsymbol{x}}\right\}_{\boldsymbol{x} \in \Omega}$ is a family of free $\mathbb{R}^{+}$-valued parameters, $\boldsymbol{U}(0, \boldsymbol{x})=\boldsymbol{U}_{\boldsymbol{x}}^{0}$ and $\boldsymbol{V}(0, \boldsymbol{x})=$ $\boldsymbol{V}_{\boldsymbol{x}}^{0}$ almost surely (a.s.) and $\left\{\boldsymbol{W}(r, \boldsymbol{x}), \boldsymbol{x} \in \Omega, r \in \mathbb{R}^{+}\right\}$is the Gaussian random field defined in $\S 2.1$. The probability distribution $P_{\boldsymbol{U}_{\boldsymbol{x}}^{0}, \boldsymbol{V}_{\boldsymbol{x}}^{0}}(\mathrm{~d} \boldsymbol{u}, \mathrm{d} \boldsymbol{v} ; \boldsymbol{x})$ of $\left(\boldsymbol{U}_{\boldsymbol{x}}^{0}, \boldsymbol{V}_{\boldsymbol{x}}^{0}\right)$ is assumed to be known. Note that a deterministic initialization vector $\left(\boldsymbol{u}_{\boldsymbol{x}}^{0}, \boldsymbol{v}_{\boldsymbol{x}}^{0}\right) \in$ $\mathbb{R}^{n} \times \mathbb{R}^{n}$ can be used, in which case $P_{\boldsymbol{U}_{\boldsymbol{x}}^{0}, \boldsymbol{V}_{\boldsymbol{x}}^{\mathrm{o}}}(\mathrm{d} \boldsymbol{u}, \mathrm{d} \boldsymbol{v} ; \boldsymbol{x})=\delta_{\mathbf{0}}\left(\boldsymbol{u}-\boldsymbol{u}_{\boldsymbol{x}}^{0}\right) \otimes \delta_{0}\left(\boldsymbol{v}-\boldsymbol{v}_{\boldsymbol{x}}^{0}\right)$, with $\delta_{\mathbf{0}}$ the Dirac measure at the origin of $\mathbb{R}^{n}$. We denote by $\left\{\boldsymbol{b}_{\boldsymbol{x}}: \mathbb{R}^{n} \times \mathbb{R}^{n} \rightarrow \mathbb{R}^{2 n}\right\}_{\boldsymbol{x} \in \Omega}$ and $\left\{\left[\sigma_{\boldsymbol{x}}\right] \in \mathbb{M}_{2 n}^{S}(\mathbb{R})\right\}_{\boldsymbol{x} \in \Omega}$ the families of drift vectors and diffusion matrices associated with the family of solution diffusion processes, defined for any $\boldsymbol{x}$ fixed in $\Omega$ as

$$
\boldsymbol{b}_{\boldsymbol{x}}(\boldsymbol{u}, \boldsymbol{v})=\left[\begin{array}{c}
\boldsymbol{v} \\
-\nabla_{\boldsymbol{u}} \Phi(\boldsymbol{u} ; \boldsymbol{x})-\frac{f_{\boldsymbol{x}}}{2} \boldsymbol{v}
\end{array}\right]
$$

and

$$
\left[\sigma_{\boldsymbol{x}}\right]=\left[a_{\boldsymbol{x}}\right]\left[a_{\boldsymbol{x}}\right]^{\mathrm{T}}, \quad\left[a_{\boldsymbol{x}}\right]=\left[\begin{array}{cc}
{\left[0_{n}\right]} & {\left[0_{n}\right]} \\
{\left[0_{n}\right]} & \sqrt{f_{\boldsymbol{x}}}\left[I_{n}\right]
\end{array}\right]
$$

for all $(\boldsymbol{u}, \boldsymbol{v}) \in \mathbb{R}^{n} \times \mathbb{R}^{n}$. We first assume that each ISDE of the family $\mathcal{F}^{\mathrm{ISDE}}$ admits a unique solution which is defined almost surely for all $r \geq 0$ and that for $r \rightarrow+\infty$, there is an asymptotic stationary solution which is denoted as $\left\{\left(\boldsymbol{U}^{\mathrm{s}}(r, \boldsymbol{x}), \boldsymbol{V}^{\mathrm{s}}(r, \boldsymbol{x})\right), r \geqslant 0\right\}$. We denote as $\left\{P^{\mathrm{s}}(\cdot, \cdot ; \boldsymbol{x})\right\}_{\boldsymbol{x} \in \Omega}$ the family of invariant measures associated with the family of stationary solutions $\left\{\left\{\left(\boldsymbol{U}^{\mathrm{s}}(r, \boldsymbol{x}), \boldsymbol{V}^{\mathrm{s}}(r, \boldsymbol{x})\right), r \geqslant 0\right\}\right\}_{\boldsymbol{x} \in \Omega}$ and introduce the family $\left\{\rho^{\mathrm{s}}(\cdot, \cdot ; \boldsymbol{x})\right\}_{\boldsymbol{x} \in \Omega}$ of probability density functions such that for $\boldsymbol{x}$ fixed in $\Omega$ :

$$
P^{\mathrm{s}}(\mathrm{d} \boldsymbol{u}, \mathrm{d} \boldsymbol{v} ; \boldsymbol{x})=\rho^{\mathrm{s}}(\boldsymbol{u}, \boldsymbol{v} ; \boldsymbol{x}) \mathrm{d} \boldsymbol{u} \mathrm{d} \boldsymbol{v} .
$$

The construction of a unique asymptotic stationary second-order solution for any $\boldsymbol{x} \in \Omega$ requires the family $\{\Phi(\cdot ; \boldsymbol{x})\}_{\boldsymbol{x} \in \Omega}$ to satisfy a few properties. Specifically, each element of the the family $\{\Phi(\cdot ; \boldsymbol{x})\}_{\boldsymbol{x} \in \Omega}$ of potential functions is assumed to satisfy the following properties, for any $\boldsymbol{x}$ fixed in $\Omega$ :

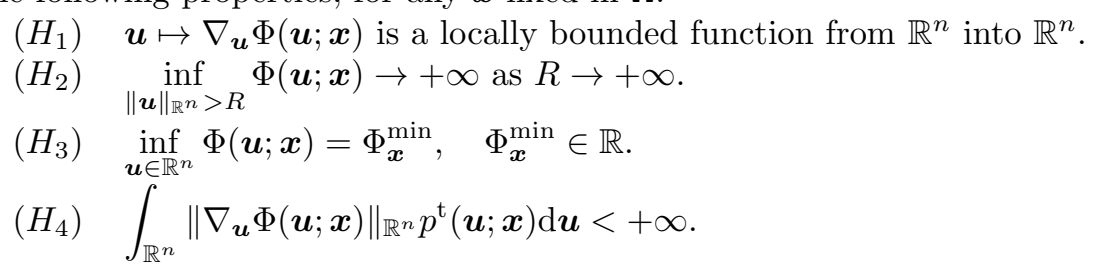


Under the above assumptions, each element $\rho^{\mathrm{s}}(\cdot ; \boldsymbol{x})$ of the family $\left\{\rho^{\mathrm{s}}(\cdot ; \boldsymbol{x})\right\}_{\boldsymbol{x} \in \Omega}$ is the unique solution of a steady-state Fokker-Planck equation [48], hence implying the existence and uniqueness of the associated invariant measure $P^{\mathrm{s}}(\cdot, \cdot ; \boldsymbol{x})$ (see Eq. (2.7)). It can further be shown ${ }^{1}[45]$ that for all $\boldsymbol{x}$ in $\Omega$ :

$$
\rho^{\mathrm{s}}(\boldsymbol{u}, \boldsymbol{v} ; \boldsymbol{x})=c_{\boldsymbol{x}} \exp \left\{-\frac{1}{2}\|\boldsymbol{v}\|^{2}-\Phi(\boldsymbol{u} ; \boldsymbol{x})\right\}, \quad \forall(\boldsymbol{u}, \boldsymbol{v}) \in \mathbb{R}^{n} \times \mathbb{R}^{n} .
$$

Therefore, one has

$$
p^{\mathrm{t}}(\boldsymbol{u} ; \boldsymbol{x})=\int_{\mathbb{R}^{n}} \rho^{\mathrm{s}}(\boldsymbol{u}, \boldsymbol{v} ; \boldsymbol{x}) \mathrm{d} \boldsymbol{v}, \quad \forall \boldsymbol{u} \in \mathbb{R}^{n}
$$

in view of Eq. (2.3). It follows that for any $\boldsymbol{x}$ fixed in $\Omega$,

$$
\lim _{r \rightarrow+\infty} \boldsymbol{U}(r, \boldsymbol{x})=\boldsymbol{A}(\boldsymbol{x})
$$

in probability distribution. It can be deduced that solving the family of ISDEs allows sampling a family $\left\{\boldsymbol{U}^{\mathrm{s}}\left(r^{*}, \boldsymbol{x}\right\}_{\boldsymbol{x} \in \Omega}\right.$ (with $r^{*}$ sufficiently large) of random variables which defines a non-Gaussian random field admitting $\left\{p^{\mathrm{t}}(\cdot ; \boldsymbol{x})\right\}_{\boldsymbol{x} \in \Omega}$ as its family of first-order m.p.d.f. and whose correlation structure results from the nonlinear transformation of the $n$ correlation functions $\left\{\Re_{1}, \ldots, \Re_{n}\right\}$.

3. Theoretical background for the constrained case. Let us now consider the general situation where $\mathcal{S}$ is any part, bounded or semi-bounded, of $\mathbb{R}^{n}$. In this case, the potentiel functions of the family $\{\Psi(\cdot ; \boldsymbol{x})\}_{\boldsymbol{x} \in \Omega}$ do not satisfy assumption $\left(H_{1}\right)$. We first provide in $\S 3.1$ a typical example where such a situation is met in practice. The proposed strategy is then outlined in $\S 3.2$. In effect, it relies on the definition of regularized potential functions, the construction of which is addressed in $\S 3.3$.

3.1. An illustrative example in stochastic linear elasticity. In the framework of uncertainty quantification, the development of PASMs has been pioneered in [44] for the class of elliptic operators, opening up the way for extensions and generalizations to various situations (such as the modeling of permeability or elasticity tensor random fields with symmetry constraints) in [9] [10] [11] [12] [13]. Such models are intended to ensure that the modeled random quantity does satisfy all the required fundamental mathematical properties (e.g. positive-definiteness) and are tailored to allow for an inverse identification through a limited parametrization. In this context, one is typically concerned with the construction of the family $\left\{p_{\boldsymbol{A}(\boldsymbol{x})}\right\}_{\boldsymbol{x} \in \Omega}$ of first-order m.p.d.f. associated with the $\mathcal{S}$-valued random field $\{\boldsymbol{A}(\boldsymbol{x}), \boldsymbol{x} \in \Omega\}$, with $^{2} \mathcal{S} \subset \mathbb{R}^{n}$. Following [44], a convenient way to achieve such a construction is to invoke the maximum entropy (MaxEnt) principle [23] [24]. The available information on random variable $\boldsymbol{A}(\boldsymbol{x})$ is then assumed to be synthesized by the following constraint (which does not include the normalization condition):

$$
\mathbb{E}\{\boldsymbol{g}(\boldsymbol{A}(\boldsymbol{x}))\}=\boldsymbol{h}_{\boldsymbol{x}}, \quad \forall \boldsymbol{x} \in \Omega,
$$

\footnotetext{
${ }^{1}$ In order to avoid any possible confusion, it is recalled here that $c_{\boldsymbol{x}}$ denotes a normalization constant whose value may vary from line to line.

${ }^{2}$ For some particular cases, one may recover a free case construction by using an alternative algebraic construction based on the exponential map; see [12] for instance.
} 
in which $\boldsymbol{a} \mapsto \boldsymbol{g}(\boldsymbol{a})$ is a measurable mapping defined from a given set $\mathbb{V} \supseteq \mathcal{S}$ into $\mathbb{R}^{m}, m \geqslant 1$, and $\left\{\boldsymbol{h}_{\boldsymbol{x}}\right\}_{\boldsymbol{x} \in \Omega}$ is a family of vectors in $\mathbb{R}^{m}$. It can be shown that the MaxEnt-based p.d.f. $\boldsymbol{a} \mapsto p_{\boldsymbol{A}(\boldsymbol{x})}(\boldsymbol{a})$ takes the form:

$$
p_{\boldsymbol{A}(\boldsymbol{x})}(\boldsymbol{a})=\mathbb{1}_{\mathcal{S}}(\boldsymbol{a}) c_{\boldsymbol{x}} \exp \left\{-\left\langle\boldsymbol{\lambda}_{\boldsymbol{x}}, \boldsymbol{g}(\boldsymbol{a})\right\rangle\right\}, \quad \forall \boldsymbol{x} \in \Omega,
$$

wherein $\boldsymbol{a} \mapsto \mathbb{1}_{\mathcal{S}}(\boldsymbol{a})$ is the indicator function for the support $\mathcal{S}$ of $p_{\boldsymbol{A}(\boldsymbol{x})}$ and $\left\{\boldsymbol{\lambda}_{\boldsymbol{x}}\right\}_{\boldsymbol{x} \in \Omega}$ is a family of vector-valued parameters defined on a subspace $\mathbb{H}_{\boldsymbol{\lambda}}$ of $\mathbb{R}^{m}$, the definition of which ensures the integrability of the p.d.f. given by Eq. (3.2). It is seen that the obtained p.d.f., defined by Eq. (3.2), takes a form that is similar (except for the presence of the indicator function) to the one stated by Eq. (2.3).

3.2. Definition of an approximating family for the non-Gaussian random field $\{\boldsymbol{A}(\boldsymbol{x}), \boldsymbol{x} \in \Omega\}$ using the free space construction. Following the above discussion, let us consider the situation where each element $p^{t}(\cdot ; \boldsymbol{x})$ of the family of prescribed marginal p.d.f. takes the form

$$
p^{\mathrm{t}}(\boldsymbol{u} ; \boldsymbol{x})=c_{\boldsymbol{x}} \exp \{-\Psi(\boldsymbol{u} ; \boldsymbol{x})\}, \quad \forall \boldsymbol{u} \in \mathcal{S},
$$

in which $\mathcal{S}$ denotes a semibounded (or bounded) subset of $\mathbb{R}^{n}$. We assume the following algebraic form (which is algebraically similar to the one derived from a MaxEnt approach; see Eq. (3.2)) for the potential function $\Psi(\cdot ; \boldsymbol{x})$ :

$$
\Psi(\boldsymbol{u} ; \boldsymbol{x})=-\log \left(\mathbb{1}_{\mathcal{S}}(\boldsymbol{u})\right)+\left\langle\boldsymbol{\lambda}_{\boldsymbol{x}}, \boldsymbol{g}(\boldsymbol{u})\right\rangle, \quad \forall \boldsymbol{u} \in \mathcal{S},
$$

with $\boldsymbol{\lambda}_{\boldsymbol{x}} \in \mathbb{H}_{\boldsymbol{\lambda}}$ a given vector and $\boldsymbol{g}: \mathbb{V} \rightarrow \mathbb{R}^{m}$ a measurable mapping. The target p.d.f. thus reads as:

$$
p^{\mathrm{t}}(\boldsymbol{u} ; \boldsymbol{x})=\mathbb{1}_{\mathcal{S}}(\boldsymbol{u}) c_{\boldsymbol{x}} \exp \left\{-\left\langle\boldsymbol{\lambda}_{\boldsymbol{x}}, \boldsymbol{g}(\boldsymbol{u})\right\rangle\right\}, \quad \forall \boldsymbol{u} \in \mathcal{S} .
$$

Because of the indicator function, the potential function defined by Eq. (3.4) cannot be readily handled by using the derivations introduced in $\S 2$ and necessitates the development of a specific methodology. For this purpose, we propose to proceed in two steps.

First of all, an additional family $\left\{\Psi_{\epsilon}(\cdot ; \boldsymbol{x})\right\}_{\boldsymbol{x} \in \Omega}$ of potential functions, with $0<$ $\epsilon \ll 1$, is introduced. Specifically, this family is such that for all $\boldsymbol{x}$ in $\Omega$, each function $\Psi_{\epsilon}(\cdot ; \boldsymbol{x})$ :

(i) Is defined by

$$
\Psi_{\epsilon}(\boldsymbol{u} ; \boldsymbol{x})=-\log \left(\mathbb{1}_{\mathcal{S}}^{\epsilon}(\boldsymbol{u})\right)+\left\langle\boldsymbol{\lambda}_{\boldsymbol{x}}, \boldsymbol{g}(\boldsymbol{u})\right\rangle, \quad \forall \boldsymbol{u} \in \mathbb{V},
$$

where $\mathbb{1}_{\mathcal{S}}^{\epsilon}$ denotes a positive regularized indicator function (defined in $\S 3.3$ ) such that $\lim _{\epsilon \downarrow 0} \mathbb{1}_{\mathcal{S}}^{\epsilon}=\mathbb{1}_{\mathcal{S}}$ and $\mathbb{V}$ is defined more precisely in the assumption below.

(ii) Satisfies hypotheses $\left(H_{1}-H_{4}\right)$.

(iii) Is such that $\lim _{\epsilon \downarrow 0} \Psi_{\epsilon}(\cdot ; \boldsymbol{x})=\Psi(\cdot ; \boldsymbol{x})$ in $\mathcal{S}^{\circ}$.

Throughout the paper, it is assumed that the family $\{\Psi(\cdot ; \boldsymbol{x})\}_{\boldsymbol{x} \in \Omega}$ satisfies the following assumption.

Assumption 1. Let $\boldsymbol{x}$ be fixed in $\Omega$. The potential function $\Psi(\cdot ; \boldsymbol{x})$ defined by Eq. (3.4) is assumed to be:

- Either repulsive on $\partial \mathcal{S}$, in which case it is further assumed that the mapping $\boldsymbol{g}$ is defined on $\mathbb{V}=\mathcal{S}^{\circ}$. 
- Or non-repulsive on $\partial \mathcal{S}$, in which case it is assumed that $\boldsymbol{g}$ is defined on $\mathbb{V}=\mathbb{R}^{n}$ and such that:

$$
\int_{\mathbb{R}^{n}} \exp \left\{-\Psi_{\epsilon}(\boldsymbol{u} ; \boldsymbol{x})\right\} \mathrm{d} \boldsymbol{u}<+\infty, \quad \forall \epsilon>0 .
$$

Let $\boldsymbol{A}_{\epsilon}(\boldsymbol{x})$ denote the random variable defined by the p.d.f.

$$
p_{\epsilon}^{\mathrm{t}}(\boldsymbol{u} ; \boldsymbol{x})=c_{\boldsymbol{x}}^{\epsilon} \exp \left\{-\Psi_{\epsilon}(\boldsymbol{u} ; \boldsymbol{x})\right\}, \quad \forall \boldsymbol{u} \in \mathbb{V},
$$

such that:

$$
\lim _{\epsilon \downarrow 0} p_{\epsilon}^{\mathrm{t}}(\cdot ; \boldsymbol{x})=p^{\mathrm{t}}(\cdot ; \boldsymbol{x}), \quad \forall \boldsymbol{x} \in \Omega .
$$

For any fixed value of $\epsilon$, we then consider solving a family $\mathcal{F}_{\epsilon}^{\text {ISDE }}$ of ISDEs whose elements read as

$$
\begin{aligned}
& \mathrm{d} \boldsymbol{U}_{\epsilon}(r, \boldsymbol{x})=\boldsymbol{V}_{\epsilon}(r, \boldsymbol{x}) \mathrm{d} r, \\
& \mathrm{~d} \boldsymbol{V}_{\epsilon}(r, \boldsymbol{x})=\left(-\nabla_{\boldsymbol{u}} \Psi_{\epsilon}\left(\boldsymbol{U}_{\epsilon}(r, \boldsymbol{x}) ; \boldsymbol{x}\right)-\frac{f_{\boldsymbol{x}}}{2} \boldsymbol{V}_{\epsilon}(r, \boldsymbol{x})\right) \mathrm{d} r+\sqrt{f_{\boldsymbol{x}}} \mathrm{d} \boldsymbol{W}(r, \boldsymbol{x}),
\end{aligned},
$$

for $r>0$ and $\boldsymbol{x}$ fixed in $\Omega$. Eq. (3.10) is supplemented with suitable initial conditions. All the parameters (initial conditions, and so on) associated with the above family $\mathcal{F}_{\epsilon}^{\text {ISDE }}$ are denoted using notations similar to those introduced in $\S 2$, except for the subscript $\epsilon$. For any fixed value of $\epsilon$ and following the derivations in $\S 2$, one has:

$$
\lim _{r \rightarrow+\infty} \boldsymbol{U}_{\epsilon}(r, \boldsymbol{x})=\boldsymbol{A}_{\epsilon}(\boldsymbol{x}), \quad \forall \boldsymbol{x} \in \Omega .
$$

Thus, the family $\left\{\boldsymbol{U}_{\epsilon}^{\mathrm{s}}\left(r^{*}, \boldsymbol{x}\right\}_{\boldsymbol{x} \in \Omega}\right.$ defines, in the stationary regime (i.e. for a sufficiently large value of $r^{*}$ ), a non-Gaussian random field $\left\{\boldsymbol{A}_{\epsilon}(\boldsymbol{x}), \boldsymbol{x} \in \Omega\right\}$ that exhibits $\left\{p_{\epsilon}^{\mathrm{t}}(\cdot ; \boldsymbol{x})\right\}_{\boldsymbol{x} \in \Omega}$ as its family of first-order m.p.d.f. The nonlinear transformation (see e.g. Eq. (1.7) for the free case) and the correlation function of the centered Gaussian random field $\left\{\boldsymbol{\Xi}(\mathbf{x}), \boldsymbol{x} \in \mathbb{R}^{d}\right\}$ completely define the system of marginal distributions of random field $\left\{\boldsymbol{A}_{\epsilon}(\boldsymbol{x}), \boldsymbol{x} \in \Omega\right\}$. When the first-order m.p.d.f. is invariant under translation in $\mathbb{R}^{d}$, this mapping is independent of $\boldsymbol{x}$ and random field $\left\{\boldsymbol{A}_{\epsilon}(\boldsymbol{x}), \boldsymbol{x} \in \Omega\right\}$ is homogeneous for the translation in $\mathbb{R}^{d}$. Finally, for all $\boldsymbol{x}$ fixed in $\Omega$, if Eq. (3.9) holds, then the sequence $\left\{\boldsymbol{A}_{\epsilon}(\boldsymbol{x})\right\}_{\epsilon>0}$ converges (in probability distribution) towards $\boldsymbol{A}(\boldsymbol{x})$ as $\epsilon \downarrow 0$.

In a second step detailed in $\S 4$, the use of an adaptivity procedure for the discretization of each ISDE is investigated. To this aim, we build on the algorithm proposed by Lemaire in [31] for the approximation of diffusion processes with nonglobally Lipschitz drift vector fields. A key feature of the algorithm (which is detailed in $\S 4$ ) is that the stochastic time step is automatically refined whenever the current state goes closer to the boundary of the admissible space, hence avoiding the use of a too small integration step that may slow down the convergence towards the stationary solution (and thus, increase the global CPU-time). At this stage, it should be noted that this paper is not concerned with the general study of some optimal adaptation mechanism or scheme for the discretization of stochastic differential equations (the optimality being understood in the sense of some error metric, e.g. a $L_{2}$ norm). The interested reader is referred to the large literature on this topic; see for instance [26] [27] [38] for the definition of algorithms in the case of scalar stochastic differential equations with an additive noise; see e.g. [28] and the references therein for a discussion and the derivation of an alternative adaptation approach controlled by the drift coefficient. 
Remark 1. The first case in Assumption 1 is especially relevant to physically sound UQ analysis, in which one is usually concerned with the modeling of random variables with values in $\mathbb{M}_{q}^{+}(\mathbb{R})$. In this case, it is usually required that the vector representations $\boldsymbol{A}(\boldsymbol{x})$ and $\boldsymbol{A}(\boldsymbol{x})^{-1}$ are both second-order random variables, that is,

$$
\mathbb{E}\left\{\|\boldsymbol{A}(\boldsymbol{x})\|^{2}\right\}<+\infty, \quad \mathbb{E}\left\{\left\|\boldsymbol{A}(\boldsymbol{x})^{-1}\right\|^{2}\right\}<+\infty, \quad \forall \boldsymbol{x} \in \Omega .
$$

It can then be shown that a sufficiently fast decrease of the probability measure near the origin $\mathbf{0} \in \partial \mathcal{S}$ of $\mathbb{R}^{n}$ is required for Eq. (3.12) to hold [43], meaning that the gradient of the potential function significantly increases in the neighbourhood of $\partial \mathcal{S}$. This important feature, combined to the additional repulsion generated by the regularized indicator function, makes the associated potential function strongly repulsive near $\partial \mathcal{S}$, so that $\boldsymbol{U}$ is trapped inside the admissible domain $\mathcal{S}$ and never reaches the boundary in practice (see $\S 5.2$ ). For $\mathbb{V}=\mathbb{R}^{n}$, the point $\boldsymbol{U}$ is allowed to go beyond $\partial \mathcal{S}$ (in a way that depends on the selected value of $\epsilon$ ) and is then pulled back into $\mathcal{S}$ in finite time by the repulsion generated by the sole regularized indicator function (see $\S 5.1$ ), hence avoiding the use of a rejection-like generation algorithm. This repulsive effect allows, through a simple particle tracking, to accomodate the case where the generator is subsequently plugged into a formulation which requires that $\boldsymbol{U} \in \mathcal{S}^{\circ}$ or $\boldsymbol{U} \in \overline{\mathcal{S}}$ almost surely at any point $\boldsymbol{x}$ in $\Omega$. The tracking procedure may for instance consists in selecting, for each simulation run, a time instant $\widetilde{r}^{*} \geqslant r^{*}$ such that $U\left(\widetilde{r}^{*}, \boldsymbol{x}\right) \in \mathcal{S}^{\circ}\left(\right.$ or $\left.U\left(\widetilde{r}^{*}, \boldsymbol{x}\right) \in \overline{\mathcal{S}}\right)$ for all $\boldsymbol{x}$ in $\Omega$ - such a strategy may, however, increase the computational time for simulations performed over large lattices.

Remark 2. Below, we briefly discuss an alternative approach following a strategy similar to that introduced above, except that the formulation is based on a classical Metropolis-Hastings algorithm [35] [25]. Assume first that the random field $\{\boldsymbol{A}(\boldsymbol{x}), \boldsymbol{x} \in \Omega\}$ under consideration has been discretized and must be accordingly sampled over a grid defined by a set $\left\{\boldsymbol{x}^{1}, \ldots, \boldsymbol{x}^{N}\right\}$ of $N$ non-overlapping points. Upon using the same generation structure as the one followed for the discretization of each ISDE (see $\S 4$ ), a Metropolis-Hastings-like generation scheme for the random field can be readily obtained as

$$
\boldsymbol{A}\left(\boldsymbol{x}^{i}\right)=\lim _{k \rightarrow+\infty} \boldsymbol{U}^{k}\left(\boldsymbol{x}^{i}\right), \quad \forall i \in\{1, \ldots, N\},
$$

where

$$
\boldsymbol{U}^{k+1}\left(\boldsymbol{x}^{i}\right)=\boldsymbol{U}^{k}\left(\boldsymbol{x}^{i}\right)+\sigma_{n}\left\{\prod_{j=1}^{N} \mathbb{1}_{\left[0 ; \min \left(1, q_{j}^{(k+1)}\right)\right]}\left(\alpha_{j}^{(k+1)}\right)\right\} \boldsymbol{\Xi}_{\boldsymbol{x}^{(i)}}\left(\theta_{k+1}\right),
$$

and $\boldsymbol{U}^{0}\left(\boldsymbol{x}^{i}\right)=\boldsymbol{u}_{\boldsymbol{x}^{i}}^{0}$, with $\boldsymbol{u}_{\boldsymbol{x}^{i}}^{0}$ an arbitrary initialization vector in $\mathcal{S}^{\circ}$. In Eq. (3.14), $\sigma_{n}>0$ denotes the standard deviation for the Gaussian transition kernel (see [39] for a discussion regarding the tuning of this parameter), $\left\{\alpha_{j}^{(k+1)}\right\}_{j=1}^{N}$ is a set of $N$ independent realizations of a random variable that is uniformly distributed over $[0,1]$ and

$$
q_{j}^{(k+1)}=\frac{p_{\boldsymbol{x}}^{\mathrm{t}}\left(\boldsymbol{U}^{k}\left(\boldsymbol{x}^{j}\right)+\sigma_{n} \boldsymbol{\Xi}_{\boldsymbol{x}^{(j)}}\left(\theta_{k+1}\right)\right)}{p_{\boldsymbol{x}}^{\mathrm{t}}\left(\boldsymbol{U}^{k}\left(\boldsymbol{x}^{j}\right)\right)}, \quad \forall j \in\{1, \ldots, N\}
$$

Besides its simplicity (for implementation in particular), the main advantage of such a strategy is that it can readily handle any type of target m.p.d.f. without requiring 
the convergence of a sequence of approximating solutions to be studied. Nevertheless, it should be pointed out that such an algorithm necessitates the acceptance of the states at all the points $\boldsymbol{x}^{1}, \ldots, \boldsymbol{x}^{N}$ (i.e. that at least, $\boldsymbol{U}^{k}\left(\boldsymbol{x}^{j}\right)+\sigma_{n} \boldsymbol{\Xi}_{\boldsymbol{x}^{(j)}}\left(\theta_{k+1}\right) \in \mathcal{S}$ for all $j$ in $\{1, \ldots, N\}$ ) for a new sample to be generated (see Eq. (3.14)), hence resulting in a cumbersome computational effort for typical values of $N$. In practice, such a scheme may thus be restricted to the case where $\mathcal{S}$ occupies a large region of $\mathbb{R}^{n}$ (so that the probability of reaching $\partial \mathcal{S}$ is reasonably small), as well as to moderate values of $N$.

3.3. Construction of the approximating family $\left\{\Psi_{\epsilon}(\cdot ; \boldsymbol{x})\right\}_{\boldsymbol{x} \in \Omega}$ of potential functions. Following $\S 3.2$, let $\{\Psi(\cdot ; \boldsymbol{x})\}_{\boldsymbol{x} \in \Omega}$ be the family of potential functions such that for all $\boldsymbol{x}$ in $\Omega$ :

$$
\Psi(\boldsymbol{u} ; \boldsymbol{x})=-\log \left(\mathbb{1}_{\mathcal{S}}(\boldsymbol{u})\right)+\left\langle\boldsymbol{\lambda}_{\boldsymbol{x}}, \boldsymbol{g}(\boldsymbol{u})\right\rangle, \quad \forall \boldsymbol{u} \in \mathcal{S} .
$$

Let $\mathbb{1}_{\mathcal{S}}^{\epsilon}$ be the regularized indicator function defined as

$$
\mathbb{1}_{\mathcal{S}}^{\epsilon}=K_{\epsilon} * \mathbb{1}_{\mathcal{S}},
$$

in which $\boldsymbol{z} \mapsto K_{\epsilon}(\boldsymbol{z})$ is a regularizing kernel on $\mathbb{R}^{n}$, parametrized by $0<\epsilon \ll 1$, and $*$ denotes the convolution operator:

$$
\mathbb{1}_{\mathcal{S}}^{\epsilon}(\boldsymbol{u})=\int_{\mathbb{R}^{n}} K_{\epsilon}(\boldsymbol{z}) \mathbb{1}_{\mathcal{S}}(\boldsymbol{u}-\boldsymbol{z}) \mathrm{d} \boldsymbol{z}, \quad \forall \boldsymbol{u} \in \mathbb{R}^{n} .
$$

Here, and without loss of generality, a regularization by a centered Gaussian kernel

$$
K_{\epsilon}(\boldsymbol{z})=\frac{1}{\epsilon^{n}(2 \pi)^{n / 2}} \exp \left\{-\frac{1}{2} \frac{\|\boldsymbol{z}\|^{2}}{\epsilon^{2}}\right\}, \quad \forall \boldsymbol{z} \in \mathbb{R}^{n},
$$

is used. It can be deduced that $\mathbb{1}_{\mathcal{S}}^{\epsilon}$ equivalently reads as

$$
\mathbb{1}_{\mathcal{S}}^{\epsilon}(\boldsymbol{u})=\mathbb{E}\left\{\mathbb{1}_{\mathcal{S}}\left(\boldsymbol{u}-\boldsymbol{Z}_{\epsilon}\right)\right\}, \quad \forall \boldsymbol{u} \in \mathbb{R}^{n},
$$

where $\boldsymbol{Z}_{\epsilon}$ is a $\mathbb{R}^{n}$-valued centered Gaussian random variable whose p.d.f. is given by the right-hand side of Eq. (3.19). The approximating family $\left\{\Psi_{\epsilon}(\cdot ; \boldsymbol{x})\right\}_{\boldsymbol{x} \in \Omega}$ of potential functions is then defined by:

$$
\Psi_{\epsilon}(\boldsymbol{u} ; \boldsymbol{x})=-\log \left(\mathbb{1}_{\mathcal{S}}^{\epsilon}(\boldsymbol{u})\right)+\left\langle\boldsymbol{\lambda}_{\boldsymbol{x}}, \boldsymbol{g}(\boldsymbol{u})\right\rangle, \quad \forall \boldsymbol{u} \in \mathbb{V} .
$$

Remark 3. Several approaches can be pursued in order to compute the indicator function $\mathbb{1}_{\mathcal{S}}^{\epsilon}$. A brute-force approach consists in evaluating the regularization given by Eq. (3.17), regardless of the current value of $\boldsymbol{u}$. From a computational standpoint, it is clear that such a strategy is certainly not optimal, unless the neighborhood of boundary $\partial \mathcal{S}$ is expected to be reached with a quite large probability. One may alternatively choose to compute the regularized function only when the state $\boldsymbol{u}$ is "sufficiently" close to $\partial \mathcal{S}$. However, one should note that the numerical expense induced by such a strategy, which requires the distance between $\boldsymbol{u}$ and its projection onto $\partial \mathcal{S}$ to be computed, is conditionned by the nature of the boundary.

4. Definition of a discretization scheme with a stochastic adaptive step sequence. For $k \geqslant 0$, we let $\boldsymbol{U}_{\epsilon}^{k}(\boldsymbol{x})=\boldsymbol{U}_{\epsilon}\left(r_{k}, \boldsymbol{x}\right), \boldsymbol{V}_{\epsilon}^{k}(\boldsymbol{x})=\boldsymbol{V}_{\epsilon}\left(r_{k}, \boldsymbol{x}\right)$ and $\boldsymbol{W}^{k}(\boldsymbol{x})=$ $\boldsymbol{W}\left(r_{k}, \boldsymbol{x}\right)$, where $\left\{r_{0}, \ldots, r_{M}\right\}$ constitutes some stochastic discretization (see $\left.\S 4.2\right)$ of 
time interval $[0 ; T]$, with $T=r_{M}$. For $\boldsymbol{x}$ fixed in $\Omega$ and $k \geqslant 0$, we denote as $\boldsymbol{L}^{k}(\boldsymbol{x})$ the $\mathbb{R}^{n}$-valued random variable defined as:

$$
\forall j \in\{1, \ldots, n\}, \quad\left(L^{k}(\boldsymbol{x})\right)_{j}=-\left\{\frac{\partial \Psi(\boldsymbol{u} ; \boldsymbol{x})}{\partial u_{j}}\right\}_{\boldsymbol{u}=\boldsymbol{U}^{k}(\boldsymbol{x})} .
$$

Similarly, $\left(L_{\epsilon}^{k}(\boldsymbol{x})\right)_{j}$ denotes the $j$-th component of the gradient of $\Psi_{\epsilon}(\cdot ; \boldsymbol{x})$ evaluated at $\boldsymbol{u}=\boldsymbol{U}_{\epsilon}^{k}(\boldsymbol{x})$. When a comparison between either numerical schemes or several parametrizations is performed (at a given point $\boldsymbol{x}$ ), we use the same initial conditions and the same sample path for the increments of the normalized Wiener processes.

4.1. Numerical comparison between the explicit Euler and StörmerVerlet schemes for the free case construction. Before proceeding to the construction of the general algorithm, we numerically compare below the convergence of the explicit Euler and Störmer-Verlet (SV) schemes, the former serving as a basis for Lemaire's derivations [31]. Details about the SV algorithm for the integration of ordinary differential equations can be found in [17] - see also [14] [16] [36] for surveys about geometric integration; see [37] and e.g. [30] for geometric integration applied to Langevin-type equations. For illustration purposes, we consider a potential function derived in [45] (with $\mathcal{S}=\mathbb{R}^{7}$ ) for the MaxEnt-based probabilistic modeling of a band $\mathbb{M}_{4}^{+}(\mathbb{R})$-valued random matrix $[\boldsymbol{A}]$.

4.1.1. Definition of the target probability distribution. Let $\boldsymbol{A}$ be the $\mathbb{R}^{7}$ valued random variable corresponding to a vector representation (see below) of the nonzero entries of $[\boldsymbol{A}]$. Let $p_{\boldsymbol{A}}$ denote its p.d.f., obtained by invoking the MaxEnt principle. Let $\boldsymbol{u} \mapsto \boldsymbol{e}(\boldsymbol{u})$ and $\boldsymbol{u} \mapsto[\mathcal{G}(\boldsymbol{u})]$ be the two unique non-linear deterministic mappings defined from $\mathbb{R}^{7}$ into $\mathbb{R}^{7}$ and from $\mathbb{R}^{7}$ into $\mathbb{M}_{7}^{+}(\mathbb{R})$ respectively, such that $\forall \boldsymbol{u} \in \mathbb{R}^{7}$,

$$
\boldsymbol{e}(\boldsymbol{u})=\left(u_{1}^{4}, u_{1}^{2} u_{2}, u_{2}^{2}+u_{3}^{4}, u_{3}^{2} u_{4}, u_{4}^{2}+u_{5}^{4}, u_{5}^{2} u_{6}, u_{6}^{2}+u_{7}^{4}\right)
$$

and $[\mathcal{G}(\boldsymbol{u})]=[\mathcal{L}(\boldsymbol{u})]^{\mathrm{T}}[\mathcal{L}(\boldsymbol{u})]$, with

$$
[\mathcal{L}(\boldsymbol{u})]=\left[\begin{array}{cccc}
u_{1}^{2} & u_{2} & 0 & 0 \\
0 & u_{3}^{2} & u_{4} & 0 \\
0 & 0 & u_{5}^{2} & u_{6} \\
0 & 0 & 0 & u_{7}^{2}
\end{array}\right] .
$$

It is assumed that the available information on random vector $\boldsymbol{A}$ takes the form:

$$
\mathbb{E}\{\boldsymbol{e}(\boldsymbol{A})\}=\underline{\boldsymbol{e}}, \quad \mathbb{E}\left\{\|[\mathcal{G}(\boldsymbol{A})]\|_{\mathrm{F}}^{2}\right\}=7.8575, \quad \mathbb{E}\left\{\left\|[\mathcal{G}(\boldsymbol{A})]^{-1}\right\|_{\mathrm{F}}^{2}\right\}=5.6,
$$

with $\underline{\boldsymbol{e}}=(1,0,1,0,1,0,1)$. The associated potential function $\Phi: \mathbb{R}^{7} \rightarrow \mathbb{R}$ such that $p_{\boldsymbol{A}}(\boldsymbol{a})=c \exp \{-\Phi(\boldsymbol{a})\}$ for all $\boldsymbol{a}$ in $\mathbb{R}^{7}$ is then given by:

$$
\Phi(\boldsymbol{u})=\left\langle\boldsymbol{\lambda}^{(1)}, \boldsymbol{e}(\boldsymbol{u})\right\rangle+\lambda^{(2)}\|[\mathcal{G}(\boldsymbol{u})]\|_{\mathrm{F}}^{2}+\lambda^{(3)}\left\|[\mathcal{G}(\boldsymbol{u})]^{-1}\right\|_{\mathrm{F}}^{2}, \quad \forall \boldsymbol{u} \in \mathbb{R}^{7} .
$$

In Eq. (4.5), the Lagrange multipliers $\lambda^{(1)} \in \mathbb{R}^{7}, \lambda^{(2)} \in \mathbb{R}_{*}^{+}$and $\lambda^{(3)} \in \mathbb{R}_{*}^{+}$are such that the constraints given by Eq. (4.4) are fullfiled and are found to be (see $[45]): \lambda^{(1)}=(0.7381,4.1697,1.2465,-0.9248,0.8998,4.2584,0.8714), \lambda^{(2)}=2.2293$ and $\lambda^{(3)}=1.7749$. 
4.1.2. Discretization schemes and numerical results. Let $\boldsymbol{U}^{1}=\boldsymbol{u}_{0}$ and $\boldsymbol{V}^{1}=\boldsymbol{v}_{0}$, where $\boldsymbol{u}_{0}$ and $\boldsymbol{v}_{0}$ are two given deterministic vectors in $\mathbb{R}^{7}$. For $k=$ $1, \ldots, M-1$, the explicit Euler scheme reads as

$$
\begin{aligned}
\boldsymbol{U}^{k+1} & =\boldsymbol{U}^{k}+\Delta r \boldsymbol{V}^{k}, \\
\boldsymbol{V}^{k+1} & =\left(1-\frac{f \Delta r}{2}\right) \boldsymbol{V}^{k}+\Delta r \boldsymbol{L}^{k}+\sqrt{f} \Delta \boldsymbol{W}^{k+1},
\end{aligned}
$$

where the increment $\Delta \boldsymbol{W}^{k+1}=\boldsymbol{W}^{k+1}-\boldsymbol{W}^{k}$ of the normalized Wiener process $\boldsymbol{W}$ is a second-order Gaussian centered $\mathbb{R}^{n}$-valued random variable with covariance matrix $\Delta r\left[I_{n}\right]$

For $k=1, \ldots, M-1$, the $\mathrm{SV}$ scheme is defined as

$$
\begin{aligned}
\boldsymbol{U}^{k+1 / 2} & =\boldsymbol{U}^{k}+\frac{\Delta r}{2} \boldsymbol{V}^{k} \\
\boldsymbol{V}^{k+1} & =\frac{1-\beta}{1+\beta} \boldsymbol{V}^{k}+\frac{\Delta r}{1+\beta} \boldsymbol{L}^{k+1 / 2}+\frac{\sqrt{f}}{1+\beta} \Delta \boldsymbol{W}^{k+1}, \\
\boldsymbol{U}^{k+1} & =\boldsymbol{U}^{k+1 / 2}+\frac{\Delta r}{2} \boldsymbol{V}^{k+1}
\end{aligned}
$$

with $\beta=f \Delta r / 4$.

In order to provide a convergence analysis of the two above schemes, we introduce the function Conv : $\mathbb{N} \rightarrow \mathbb{R}^{+}$defined as:

$$
\operatorname{Conv}\left(M_{c}\right)=\frac{1}{M_{c}} \sum_{k=1}^{M_{c}}\left\|\boldsymbol{U}^{k}\right\|^{2}, \quad \forall M_{c} \geqslant 1 .
$$

This function measures the convergence of the ergodic estimator for the second-order moment of $\left\|\boldsymbol{U}^{k}\right\|$. We then consider the following error function ErrConv $: \mathbb{N} \rightarrow \mathbb{R}^{+}$:

$$
\operatorname{Err} \operatorname{Conv}\left(M_{c}\right)=\left|\operatorname{Conv}\left(M_{c}\right)-m_{2}^{\mathrm{ref}}\right| / m_{2}^{\mathrm{ref}} \quad \forall M_{c} \geqslant 1
$$

in which $m_{2}^{\text {ref }}$ denotes the reference value for the second-order moment of $\boldsymbol{U}$. In practice, such a value is obtained by using the explicit Euler scheme (with a sufficiently small time step, e.g. $\Delta r=10^{-5}$ ) and an ergodic estimator over a large integration interval (typically $M_{c}=10^{9}$ ). The precision and rate of convergence (towards the stationary solution) are then illustrated below by studying the convergence of mapping $M_{c} \mapsto \operatorname{ErrConv}\left(M_{c}\right)$. This convergence is displayed in Fig. 4.1 for several values of the integration step $\Delta r$ and for the two different schemes defined by Eqs. (4.6) and (4.8). As expected, it is seen that for large values of $\Delta r$, the Störmer-Verlet scheme yields a better approximation than the explicit Euler one, whereas the approximations coincide for a sufficiently small time step. These results, together with other numerical evidences which are not reported here for brevity, motivate the combination of Lemaire's adaptivity strategy with the SV scheme for the random field case.

4.2. Construction of an adaptive Störmer-Verlet scheme. The aim of this section is to propose an adaptive discretization algorithm for solving the family $\mathcal{F}_{\epsilon}^{\text {ISDE }}$ of ISDEs (the parameter $\epsilon$ being fixed) introduced in $\S 3.2$. This scheme involves (i) a specific definition of the family of Wiener increments (in accordance with $\S 2.1$ ) and (ii) a stochastic step sequence which essentially depends on two different ingredients, namely: 

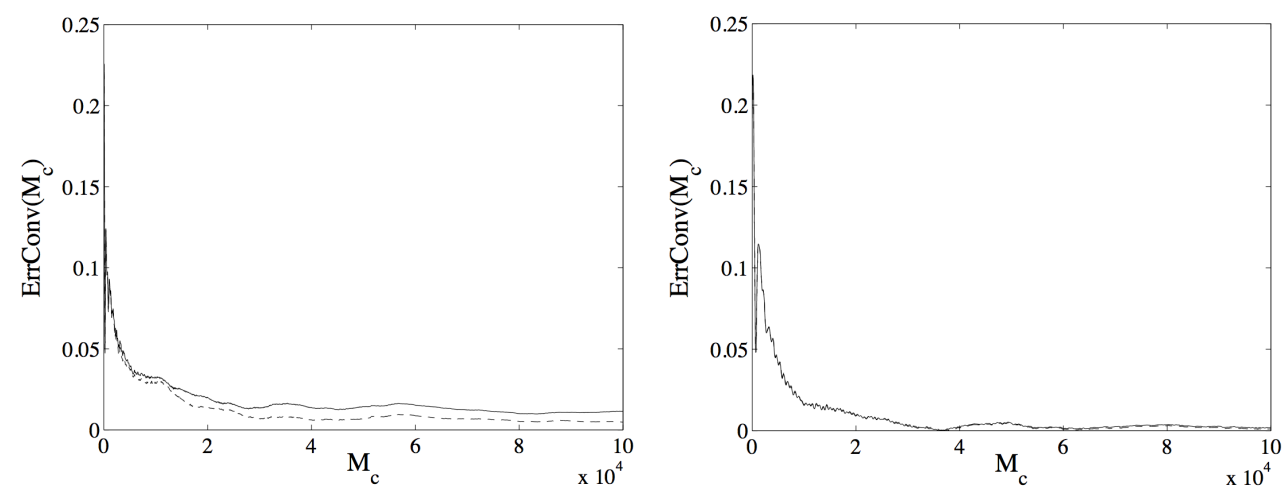

FIG. 4.1. Convergence analysis: graph of the mapping $M_{c} \mapsto \operatorname{ErrConv}\left(M_{c}\right)$ for $\Delta r=1.5 \times 10^{-3}$ (left panel) and $\Delta r=0.5 \times 10^{-3}$ (right panel). Solid line: explicit Euler scheme. Dashed line: Störmer-Verlet scheme.

- a dominating deterministic nonincreasing step sequence, which is used whenever the current state is "far enough" from $\partial \mathcal{S}$.

- a stochastic counterpart that prevents the scheme from diverging and controls the adaptivity procedure as the particle reaches the neighbourhood of the boundary $\partial \mathcal{S}$.

Following [31], let $\left(\gamma_{k}\right)_{k \geq 1}$ be a positive nonincreasing sequence such that:

$$
\gamma_{k}=\gamma_{0} k^{-1 / \tau}, \quad \forall k \geq 1,
$$

where $\gamma_{0}>0$ is a parameter corresponding to the initial time step and $\tau \in \mathbb{N}_{*}$ is a free parameter controlling the rate of decay for the deterministic step sequence (see the remark below). From Eq. (4.13), it is readily seen that

$$
\lim _{k \rightarrow+\infty} \gamma_{k}=0
$$

and

$$
\sum_{k \geq 1} \gamma_{k}=+\infty
$$

Furthermore, we introduce a family of stochastic processes $\left\{\left\{\chi_{\boldsymbol{x}}^{k}, k \geq 1\right\}\right\}_{\boldsymbol{x} \in \Omega}$ defined for all $\boldsymbol{x}$ as

$$
\chi_{\boldsymbol{x}}^{k}=\frac{2 \mathcal{V}_{\boldsymbol{x}}\left(\boldsymbol{U}_{\epsilon}^{k}(\boldsymbol{x}), \boldsymbol{V}_{\epsilon}^{k}(\boldsymbol{x})\right)}{\| \boldsymbol{b}_{\boldsymbol{x}}\left(\boldsymbol{U}_{\epsilon}^{k}(\boldsymbol{x}), \boldsymbol{V}_{\epsilon}^{k}(\boldsymbol{x}) \|^{2} \vee 1\right.}, \quad \forall k \geq 1 .
$$

In Eq. (4.16), $\mathcal{V}_{\boldsymbol{x}}$ is the Lyapunov function defined at point $\boldsymbol{x}$ as [34]

$$
\mathcal{V}_{\boldsymbol{x}}(\boldsymbol{u}, \boldsymbol{v})=\mathcal{H}_{\boldsymbol{x}}(\boldsymbol{u}, \boldsymbol{v})+\frac{f_{\boldsymbol{x}}}{4}\langle\boldsymbol{u}, \boldsymbol{v}\rangle+\frac{f_{\boldsymbol{x}}{ }^{2}}{16}\|\boldsymbol{u}\|^{2}+1, \quad \forall(\boldsymbol{u}, \boldsymbol{v}) \in \mathbb{V} \times \mathbb{R}^{n},
$$

in which $\mathcal{H}_{x}$ is the following Hamiltonian:

$$
\mathcal{H}_{\boldsymbol{x}}(\boldsymbol{u}, \boldsymbol{v})=\|\boldsymbol{v}\|^{2} / 2+\Psi_{\epsilon}(\boldsymbol{u} ; \boldsymbol{x}), \quad \forall(\boldsymbol{u}, \boldsymbol{v}) \in \mathbb{V} \times \mathbb{R}^{n}
$$


The stochastic step sequence $\left(\widetilde{\gamma}_{k+1}\right)_{k \geq 0}$ is then defined as

$$
\widetilde{\gamma}_{k+1}=\min _{\boldsymbol{x} \in \Omega}\left(\gamma_{k+1} \wedge \chi_{\boldsymbol{x}}^{k}\right), \quad \forall k \geq 1,
$$

with $\widetilde{\gamma}_{0}=\gamma_{0}$. For any $\boldsymbol{x}$ in $\Omega$ and $k=1, \ldots, M-1$, the adaptive explicit Störmerverlet (ASV) scheme then reads as:

$$
\begin{aligned}
\boldsymbol{U}_{\epsilon}^{k+1 / 2}(\boldsymbol{x}) & =\boldsymbol{U}_{\epsilon}^{k}(\boldsymbol{x})+\frac{\widetilde{\gamma}_{k+1}}{2} \boldsymbol{V}_{\epsilon}^{k}(\boldsymbol{x}), \\
\boldsymbol{V}_{\epsilon}^{k+1}(\boldsymbol{x}) & =\frac{1-\beta_{\boldsymbol{x}}}{1+\beta_{\boldsymbol{x}}} \boldsymbol{V}_{\epsilon}^{k}(\boldsymbol{x})+\frac{\widetilde{\gamma}_{k+1}}{1+\beta_{\boldsymbol{x}}} \boldsymbol{L}_{\epsilon}^{k+1 / 2}(\boldsymbol{x})+\frac{\sqrt{f_{\boldsymbol{x}}}}{1+\beta_{\boldsymbol{x}}} \Delta \boldsymbol{W}^{k+1}(\boldsymbol{x}), \\
\boldsymbol{U}_{\epsilon}^{k+1}(\boldsymbol{x}) & =\boldsymbol{U}_{\epsilon}^{k+1 / 2}(\boldsymbol{x})+\frac{\widetilde{\gamma}_{k+1}}{2} \boldsymbol{V}_{\epsilon}^{k+1}(\boldsymbol{x}),
\end{aligned}
$$

with $\beta_{\boldsymbol{x}}=f_{\boldsymbol{x}} \widetilde{\gamma}_{k+1} / 4$. For any $\boldsymbol{x}$ fixed in $\Omega$ and following $\S 2.1$, the increment of the normalized Wiener process $\{\boldsymbol{W}(r, \boldsymbol{x}), r \geqslant 0\}$ between time instances $r_{k}$ and $r_{k+1}$ is finally defined as:

$$
\Delta \boldsymbol{W}^{k+1}(\boldsymbol{x})=\boldsymbol{W}^{k+1}(\boldsymbol{x})-\boldsymbol{W}^{k}(\boldsymbol{x})=\left(\widetilde{\gamma}_{k+1}\right)^{1 / 2} \boldsymbol{\Xi}_{\boldsymbol{x}}\left(\theta_{k+1}\right) .
$$

It is worth noticing that such a definition allows spatial dependencies to be introduced while solving the family of ISDEs and is therefore well-adapted to the generation of the class of random field prior models.

Remark 4. From Eq. (4.13), it is seen that setting a large value of $\tau$ amounts to consider a very slowly decreasing deterministic counterpart in the step sequence. In effect, this allows for a fast exploration of the admissible space $\mathcal{S}$, but might slow down the convergence towards the stationary regime when the neighborhood of $\partial \mathcal{S}$ is reached with moderate to large probabilities. Conversely, retaining a small value of $\tau$ decreases the number of stochastic refinements (in the transient regime in particular) and yields a better (asymptotic) approximation of the invariant measure [29].

5. Numerical experiments. In this section, we illustrate the proposed approach through three applications. The first two applications do not address the random field case (the spatial indexation is then temporarily dropped in $\S 5.1$ and $\S 5.2)$ and specifically investigate the behavior of the algorithm for two different kinds of supports. An application to the random field case is finally provided in $\S 5.3$, where a homogeneous system of first-order m.p.d.f. is considered.

Assuming that the random field is sampled at $N$ non-overlapping points $\boldsymbol{x}^{(1)}, \ldots$, $\boldsymbol{x}^{(N)}$, let Conv be the following function:

$$
\operatorname{Conv}\left(M_{c}, \boldsymbol{x}^{(i)} ; \epsilon\right)=\frac{1}{\sum_{k=M_{0}}^{M_{c}} \widetilde{\gamma}_{k}} \sum_{k=M_{0}}^{M_{c}} \widetilde{\gamma}_{k}\left\|\boldsymbol{U}_{\epsilon}^{k-1}\left(\boldsymbol{x}^{(i)}\right)\right\|^{2}, \quad \forall M_{c} \geqslant 1,
$$

where $M_{0} \geqslant 1$ may be selected to speed up the convergence by removing the transient part in the ergodic estimator. For an homogeneous system of first-order m.p.d.f. and when a reference value $m_{2}^{\text {ref }}$ for the second-order moment of $\boldsymbol{U}$ is known, the convergence is characterized by using the following error function $M_{c} \mapsto \operatorname{ErrConv}\left(M_{c} ; \epsilon\right)$ :

$$
\operatorname{ErrConv}\left(M_{c} ; \epsilon\right)=\max _{1 \leqslant i \leqslant N}\left|\operatorname{Conv}\left(M_{c}, \boldsymbol{x}^{(i)} ; \epsilon\right)-m_{2}^{\mathrm{ref}}\right| / m_{2}^{\mathrm{ref}} \quad \forall M_{c} \geqslant 1,
$$

with Conv given by Eq. (5.1).

All routines are programmed in C, making use of the FFTW library [19] for FFT computations. The underlying random generator is the 64-bit Mersenne Twister generator from Matsumoto and Nishimura [33]. 


\subsection{Application 1.}

5.1.1. Problem setting. In this first application, we consider a two-dimensional case, $n=2$, and define the target p.d.f. $p^{\mathrm{t}}$ as

$$
p^{\mathrm{t}}(\boldsymbol{u})=c \exp \{-\Psi(\boldsymbol{u})\}, \quad \forall \boldsymbol{u} \in \mathcal{S},
$$

in which the potential function is given by

$$
\Psi(\boldsymbol{u})=-\log \left(\mathbb{1}_{\mathcal{S}}(\boldsymbol{u})\right)+\frac{1}{2}\langle[K] \boldsymbol{u}, \boldsymbol{u}\rangle, \quad \forall \boldsymbol{u} \in \mathcal{S},
$$

with $\mathcal{S}=[-0.5,0.5] \times[-0.5,0.5]$ and $[K]=\operatorname{diag}(1,1) ;$ hence, $\mathbb{V}=\mathbb{R}^{2}$. Such a potential function corresponds (except for the normalization constant) to the restriction to $\mathcal{S}$ of a $\mathbb{R}^{2}$-valued Gaussian random variable with a null mean vector and with covariance matrix $\left[I_{2}\right]$. We then introduce the approximation $\Psi_{\epsilon}$ of $\Psi$ such that

$$
\Psi_{\epsilon}(\boldsymbol{u})=-\log \left(\mathbb{1}_{\mathcal{S}}^{\epsilon}(\boldsymbol{u})\right)+\frac{1}{2}\langle[K] \boldsymbol{u}, \boldsymbol{u}\rangle, \quad \forall \boldsymbol{u} \in \mathbb{R}^{2},
$$

where the regularized indicator function $\mathbb{1}_{\mathcal{S}}^{\epsilon}$ is defined by Eq. (3.17). The graph of $\boldsymbol{u} \mapsto \mathbb{1}_{\mathcal{S}}^{\epsilon}(\boldsymbol{u})$ is displayed in Fig. 5.1 for $\epsilon=0.01$ (left panel) and $\epsilon=0.04$ (right panel).
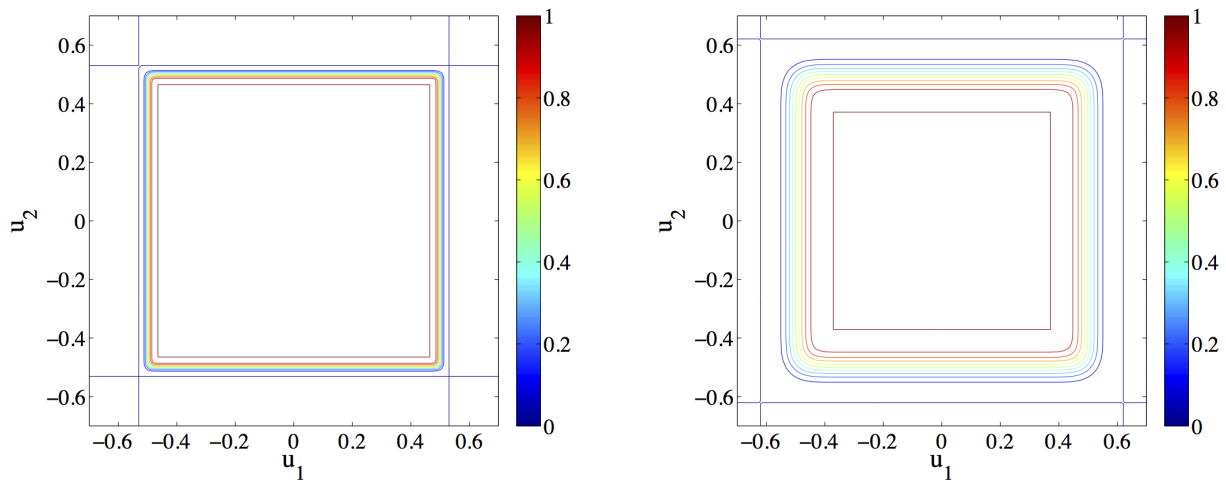

FIG. 5.1. Contour plots of the regularized indicator function $\boldsymbol{u} \mapsto \mathbb{1}_{\mathcal{S}}^{\epsilon}(\boldsymbol{u})$ for $\epsilon=0.01$ (left panel) and $\epsilon=0.04$ (right panel).

5.1.2. Numerical results. Unless otherwise stated, all the results below have been obtained by setting $\gamma_{0}=2^{-8}$ and $\tau=10$. The graph of the error function $n \mapsto \operatorname{ErrConv}(n)$ is shown in Fig. 5.2 for $\epsilon \in\{0.01,0.04,0.07,0.1\}$. It is first observed that the convergence towards the stationary solution is reached for $M_{c} \geqslant 5 \times 10^{5}$, regardless of the value of parameter $\epsilon$. As expected, the relative error decreases as $\epsilon \downarrow 0$ and is typically less than $5 \%$ for $\epsilon \leqslant 0.04$. Figure 5.3 displays a sample path of $k \mapsto\left(U_{1}^{k}, U_{2}^{k}\right)$ for $k \in \llbracket 5 \times 10^{5} ; 6 \times 10^{5} \rrbracket$ and shows how the particle of coordinates $\left(U_{1}^{k}, U_{2}^{k}\right)$ (at iteration $k$ ) fills out the domain (with $\epsilon=0.01$ ). Figure 5.4 shows a sample path $k \mapsto \widetilde{\gamma}^{k}$ of the adaptive integration step for $k \in \llbracket 5 \times 10^{5} ; 6 \times 10^{5} \rrbracket$ and $k \in \llbracket 5.013 \times 10^{5} ; 5.017 \times 10^{5} \rrbracket$; a graph of a sample path for $k \mapsto\left(U_{1}^{k}, U_{2}^{k}, \widetilde{\gamma}^{k}\right)$ is shown in Fig. $5.5(\epsilon=0.01)$. These graphs illustrate how the integration step is automatically refined (on the fly) each time the particle goes closer to the boundary 


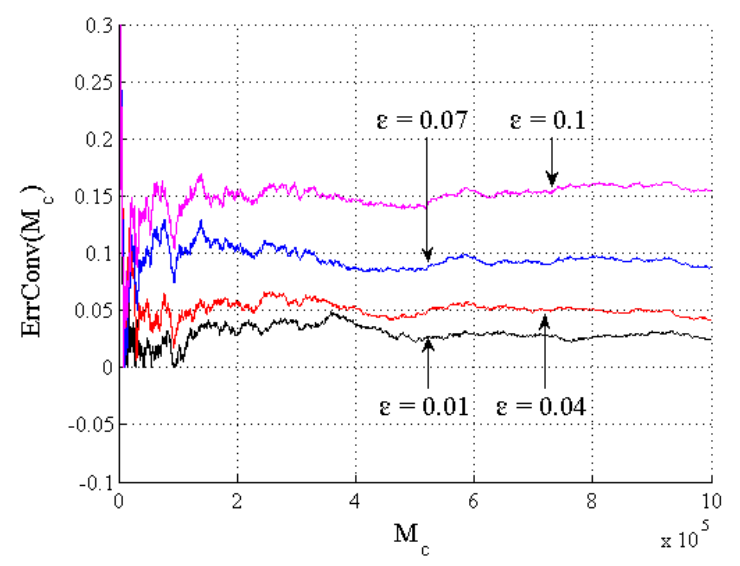

FIG. 5.2. Convergence analysis: graph of the mapping $M_{c} \mapsto \operatorname{ErrConv}\left(M_{c}\right)$ for several values of parameter $\epsilon, \gamma^{0}=2^{-8}$ and $\tau=10$. Black line: $\epsilon=0.01$; red line: $\epsilon=0.04$; blue line: $\epsilon=0.07$; magenta line: $\epsilon=0.1$.

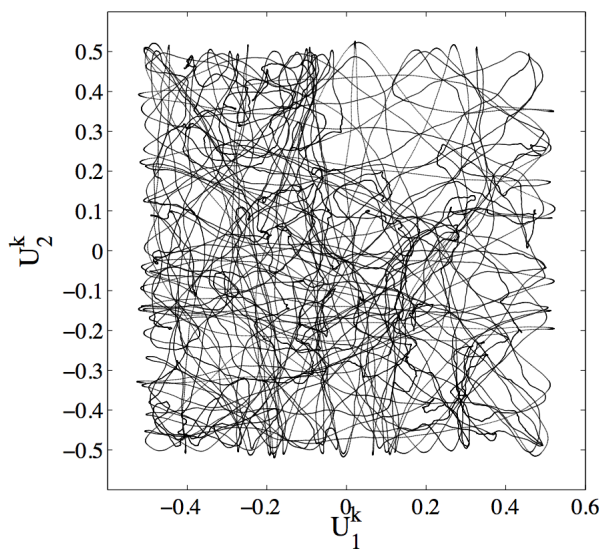

FIG. 5.3. Illustration: sample path of $k \mapsto\left(U_{1}^{k}, U_{2}^{k}\right)$ for $k \in \llbracket 5 \times 10^{5} ; 6 \times 10^{5} \rrbracket$.

of the admissible space. Specifically, the adaptive step is refined 36,410 times in average, out of one million iterations (the average being estimated from a set of 1,000 independent realizations). Note that the number of refinements does not change that much from one realization to another. A sample path for $k \mapsto\left(U_{1}^{k}, U_{2}^{k}, \widetilde{\gamma}^{k}\right)$ for $\epsilon=0.04$ and $\epsilon=0.01$ is displayed in Fig. 5.6 for a large integration range, hence showing the incidence of this parameter. The graphs of the p.d.f. $p_{U_{1}}$ of random variable $U_{1}$ (in the stationary regime) are finally shown in Figs. 5.7 and 5.8 for different values of parameter $\epsilon$, hence illustrating the convergence of the sequence as $\epsilon \downarrow 0$.

\subsection{Application 2.}

5.2.1. Problem setting. This second illustration is concerned with a regularization on the matrix set $\mathbb{M}_{q}^{+}(\mathbb{R})$. Specifically, we consider sampling from the random matrix ensemble $\mathrm{SG}^{+}$constructed in [43] (for which a reference generator is available). 

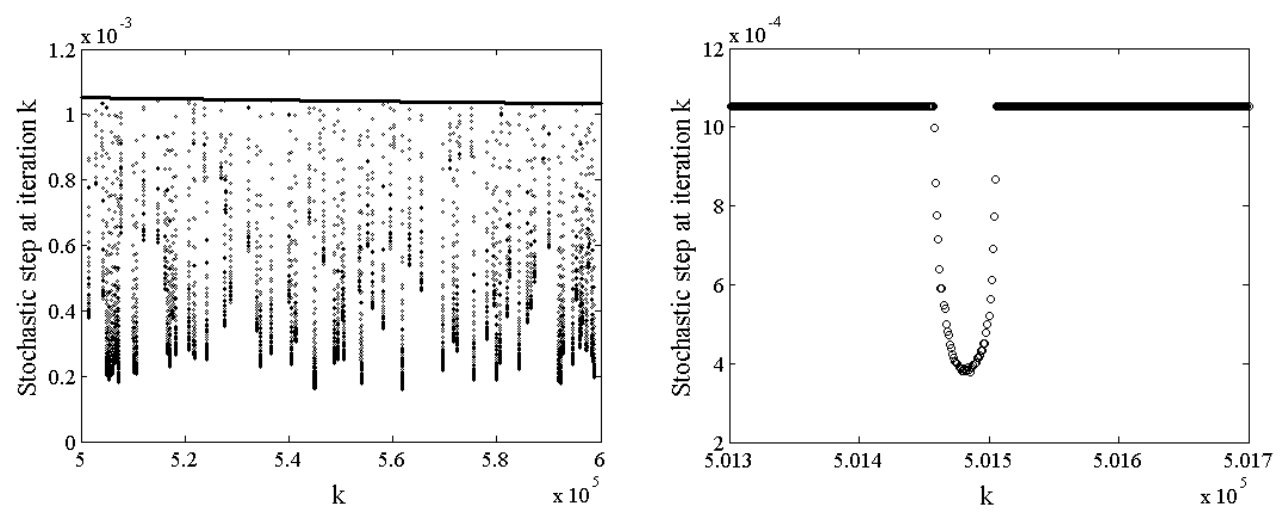

FIG. 5.4. Illustration of the adaptivity procedure: sample path of $k \mapsto \widetilde{\gamma}_{k}$ for $k \in \llbracket 5 \times 10^{5} ; 6 \times 10^{5} \rrbracket$ (left panel) and $k \in \llbracket 5.013 \times 10^{5} ; 5.017 \times 10^{5} \rrbracket$ (right panel).

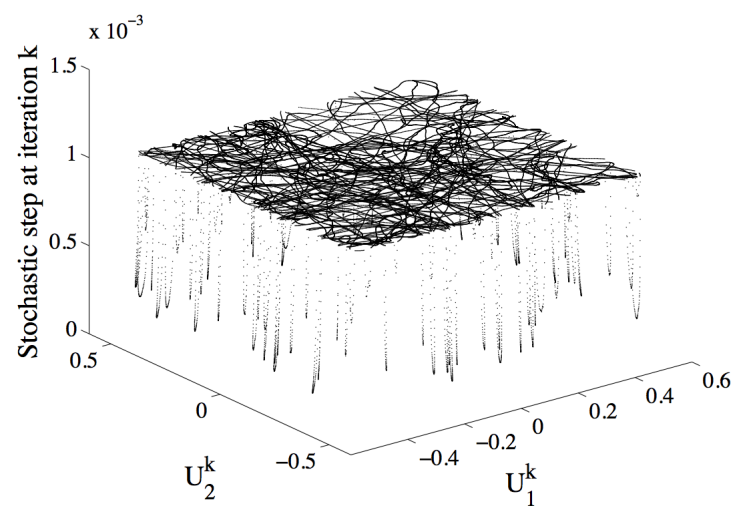

FIG. 5.5. Illustration of the adaptivity procedure: sample path of $k \mapsto\left(U_{1}^{k}, U_{2}^{k}, \widetilde{\gamma}_{k}\right)$ for $k \in$ $\llbracket 5 \times 10^{5} ; 6 \times 10^{5} \rrbracket$.
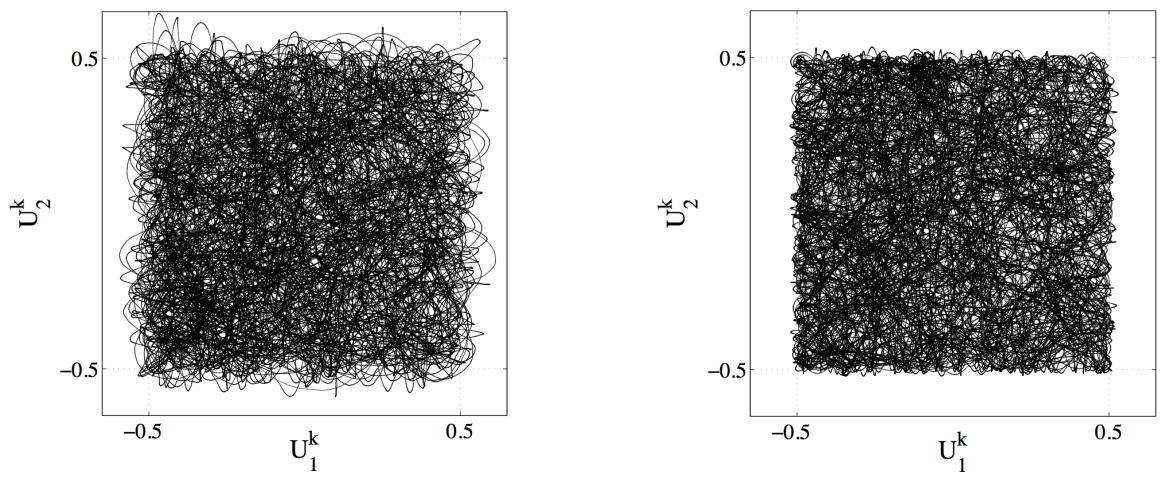

FIG. 5.6. Illustration: sample path of $k \mapsto\left(U_{1}^{k}, U_{2}^{k}\right)$ for $k \in \llbracket 5 \times 10^{5} ; 1 \times 10^{6} \rrbracket$ for $\epsilon=0.04$ (left panel) and $\epsilon=0.01$ (right panel). 

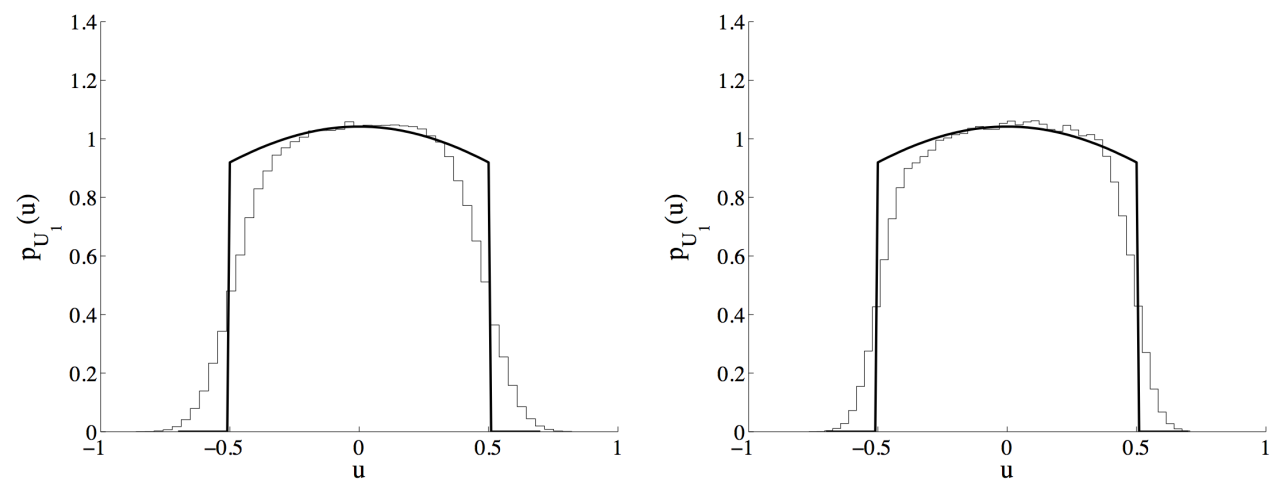

FIG. 5.7. Convergence analysis: graph of the p.d.f. $U \mapsto p_{U_{1}}(U)$ for $\epsilon=0.1$ (left panel) and $\epsilon=0.07$ (right panel): theoretical p.d.f. (thick line) and ergodic estimator (thin line).
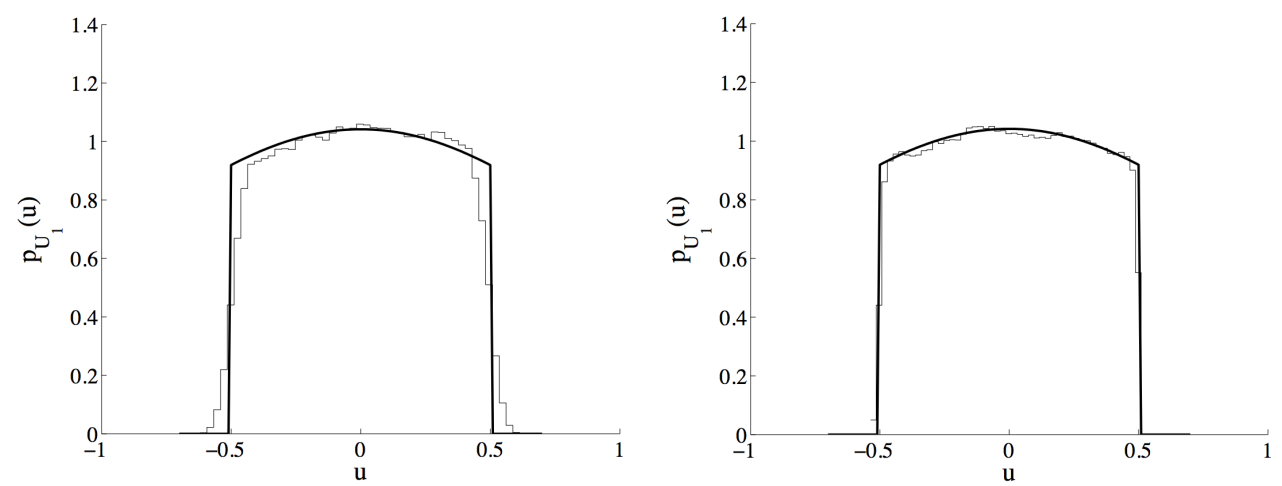

FIG. 5.8. Convergence analysis: graph of the p.d.f. $U \mapsto p_{U_{1}}(U)$ for $\epsilon=0.04$ (left panel) and $\epsilon=0.01$ (right panel): theoretical p.d.f. (thick line) and ergodic estimator (thin line).

Let $[\boldsymbol{G}] \in \mathrm{SG}^{+}$and $n=q(q+1) / 2$. The target p.d.f. $p$ of $[\boldsymbol{G}]$ is then defined as (see the reference above)

$$
p([G])=c \exp \{-\psi([G])\} \quad \forall[G] \in \mathbb{M}_{q}^{+}(\mathbb{R}),
$$

where the potential function $\psi: \mathbb{M}_{q}^{+}(\mathbb{R}) \rightarrow \mathbb{R}$ is given by

$$
\psi([G])=-\log \left(\mathbb{1}_{\mathbb{M}_{q}^{+}(\mathbb{R})}([G])\right)-(\lambda-1) \log (\operatorname{det}([G]))+\frac{q-1+2 \lambda}{2} \operatorname{tr}([G])
$$

for all $[G]$ in $\mathbb{M}_{q}^{+}(\mathbb{R})$. In Eq. (5.7), the scalar parameter $\lambda>0$ can be shown to control the level of statistical fluctuations of the random matrix $[\boldsymbol{G}]$. Let Mat : $\mathbb{R}^{n} \supset$ $\mathcal{S} \rightarrow \mathbb{M}_{q}^{+}(\mathbb{R})$ be the so-called vector-matrix mapping such that for all $[G]$ in $\mathbb{M}_{q}^{+}(\mathbb{R})$, $\exists \boldsymbol{u} \in \mathcal{S}$ such that $[G]=[\operatorname{Mat}(\boldsymbol{u})]$ with

$$
[\operatorname{Mat}(\boldsymbol{u})]=\left[\begin{array}{cccc}
u_{1} & u_{2} & \ldots & u_{q(q-1) / 2+1} \\
& u_{3} & & \vdots \\
\operatorname{sym} . & & \ddots & \\
& & & u_{n}
\end{array}\right] .
$$


The admissible space $\mathcal{S} \subset \mathbb{R}^{n}$ is thus defined as:

$$
\mathcal{S}=\left\{\boldsymbol{u} \in \mathbb{R}^{n} \mid[\operatorname{Mat}(\boldsymbol{u})] \in \mathbb{M}_{q}^{+}(\mathbb{R})\right\}
$$

It follows that the potential function $\Psi: \mathcal{S} \rightarrow \mathbb{R}$ can be written as

$$
\Psi(\boldsymbol{u})=-\log \left(\mathbb{1}_{\mathcal{S}}(\boldsymbol{u})\right)+\widehat{g}(\boldsymbol{u})
$$

with $\widehat{g}: \mathcal{S} \rightarrow \mathbb{R}$ such that

$$
\widehat{g}(\boldsymbol{u})=-(\lambda-1) \log (\operatorname{det}([\operatorname{Mat}(\boldsymbol{u})]))+\frac{q-1+2 \lambda}{2} \sum_{k=1}^{q} u_{k(k+1) / 2}
$$

The approximation $\Psi_{\epsilon}$ of $\Psi$, parametrized by $0<\epsilon \ll 1$, is then given by:

$$
\Psi_{\epsilon}(\boldsymbol{u})=-\log \left(\mathbb{1}_{\mathcal{S}}^{\epsilon}(\boldsymbol{u})\right)+\widehat{g}(\boldsymbol{u}), \quad \forall \boldsymbol{u} \in \mathcal{S}
$$

For numerical purposes, we let $q=2(n=3), \lambda=3.67$ and $f=10$. This value of $\lambda$ corresponds to a large level of statistical fluctuations, that is $\delta=0.6$.

5.2.2. Numerical results. The graph of the error function $n \mapsto \operatorname{ErrConv}(n)$ is first displayed in Fig. 5.9 for $\epsilon=0.01, \gamma^{0}=2^{-6}$ and $\tau=10^{6}$. For this sample path, 223 refinements are performed, out of 80,000 iterations. It is seen that the convergence

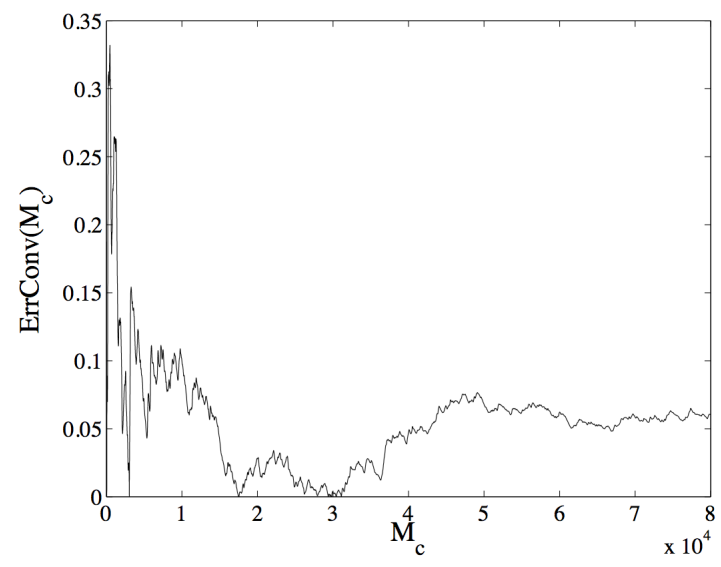

FIG. 5.9. Convergence analysis: graph of the mapping $M_{c} \mapsto \operatorname{Err} \operatorname{Conv}\left(M_{c}\right)$ for $\epsilon=0.01$, $\gamma^{0}=2^{-6}$ and $\tau=10^{6}$.

towards the stationary solution is reached for $M_{c} \geqslant 60,000$, with a relative error that is less than $6 \%$. For any $k \geqslant 1$, we further introduce the $\mathbb{R}_{*}^{+}$-valued random variable $\rho^{k}$ such that

$$
\rho^{k}=\min \mathfrak{S}\left(\left[\operatorname{Mat}\left(\boldsymbol{U}^{k}\right)\right]\right)
$$

with $\mathfrak{S}([A])$ the spectrum of $[A] \in \mathbb{M}_{q}^{S}(\mathbb{R})$. At the $k$-th iteration, $\rho^{k}$ is used to quantitatively characterize in part the distance to the boundary $\partial \mathcal{S}$. Sample paths of $k \mapsto \rho^{k}$ and $k \mapsto \widetilde{\gamma}^{k}$ are displayed in Fig. 5.10. As for the first application, it is readily observed that the step is properly refined whenever the current state 


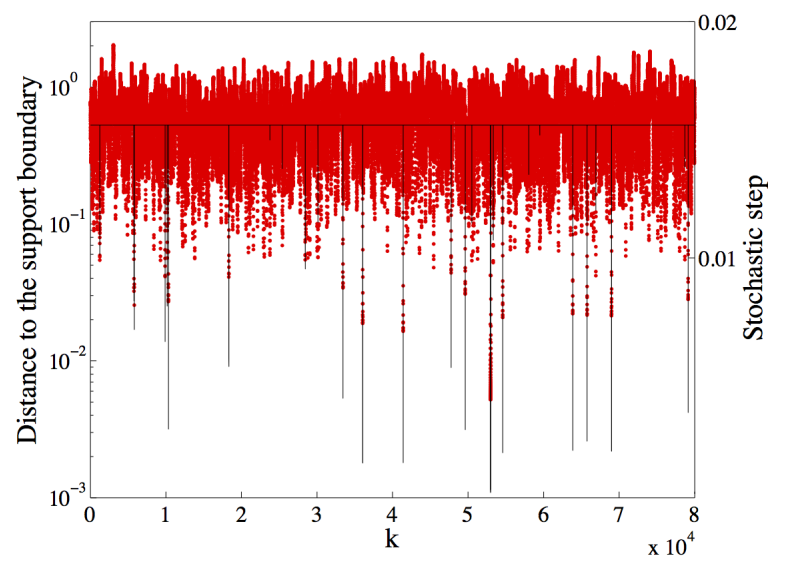

FIG. 5.10. Illustration of the adaptivity procedure: sample paths of $k \mapsto \rho^{k}$ (red circles) - left vertical axis - and $k \mapsto \widetilde{\gamma}^{k}$ (black solid line) - right vertical axis - for $\epsilon=0.01, \gamma^{0}=2^{-6}$ and $\tau=10^{6}$.

gets closer to the boundary of the admissable space $\mathcal{S}$. Further, it is seen that the probability of reaching the boundary $\partial \mathcal{S}$ turns out to be small, with only $1.6 \%$ of the steps being refined for $\epsilon=0.01$ : this property stems from the repulsion of the potential function in the neighbourhood of $\partial \mathcal{S}$, as discussed in $\S 3.2$. The convergence of the proposed algorithm is illustrated in Figs. 5.11, 5.12 and 5.13, where the firstand second-order marginal p.d.f. estimated with both the proposed and reference generators are displayed. Whereas an overall good match is observed, regardless of the random variables involved, some small discrepancies still remain for the second order marginal distributions. This fact may be due to an undersampling of the joint distributions, which are estimated from a set of 60,000 independent realizations.
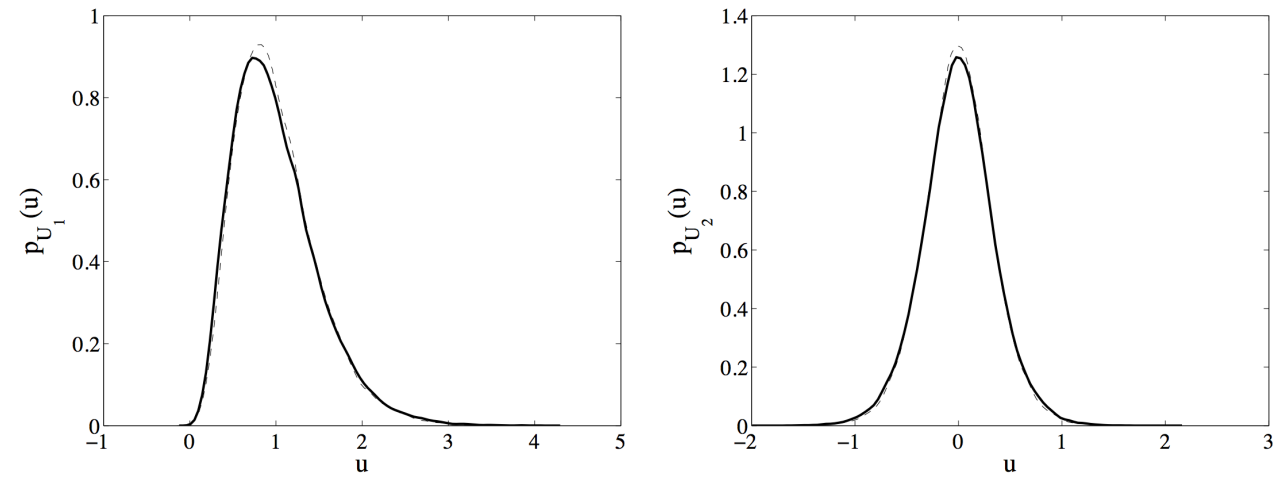

FIG. 5.11. Graph of p.d.f. $u \mapsto p_{U_{1}}(u)$ (left panel) and $u \mapsto p_{U_{2}}(u)$ (right panel): reference algorithm (solid line) and proposed approach (dashed line).

5.3. Application 3. In this section, we finally exemplify the algorithm on the $\mathbb{M}_{q}^{+}(\mathbb{R})$-valued random field whose first-order marginal p.d.f. is defined through the potential function given by Eq. (5.12). For simplicity, a two-dimensional domain $\Omega=$ (] $0,10[)^{2}$ is considered and the correlation function $\left[R_{\Xi}\right]$ of the stationary Gaussian 

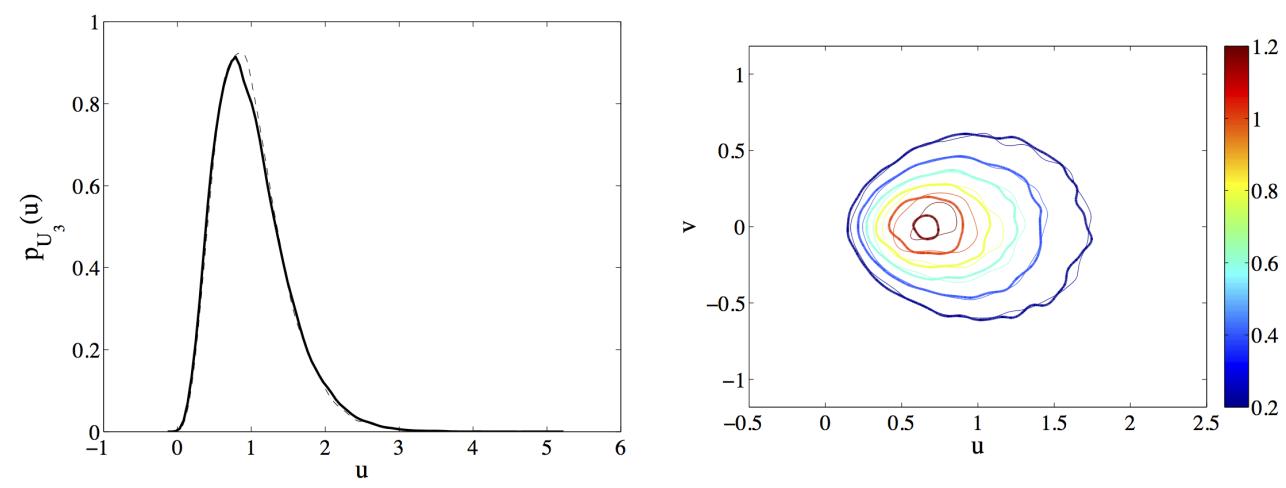

Fig. 5.12. Graph of p.d.f. $u \mapsto p_{U_{3}}(u)$ (left panel) and contour plot of $(u, v) \mapsto p_{U_{1} U_{2}}(u, v)$ (right panel): reference algorithm (solid/thick line(s)) and proposed approach (dashed/thin line(s)).
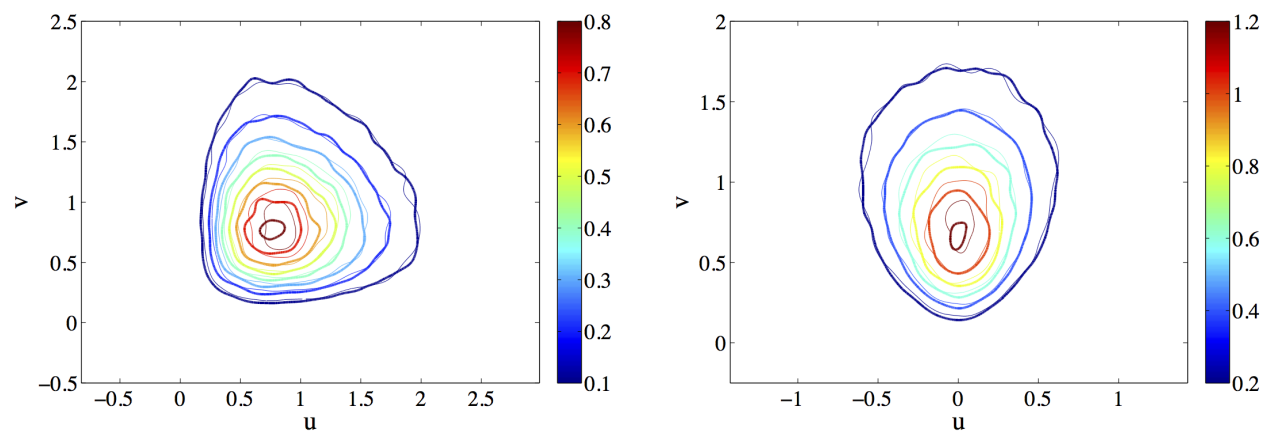

FIG. 5.13. Contour plots of $(u, v) \mapsto p_{U_{1} U_{3}}(u, v)$ (left panel) and $(u, v) \mapsto p_{U_{2} U_{3}}(u, v)$ (right panel): reference algorithm (thick lines) and proposed approach (thin lines).

random field $\left\{\boldsymbol{\Xi}(\boldsymbol{x}), \boldsymbol{x} \in \mathbb{R}^{d}\right\}$ (see $\left.\S 1.2\right)$ is defined by setting:

$$
\forall\left(\boldsymbol{x}, \boldsymbol{x}^{\prime}\right) \in \mathbb{R}^{d} \times \mathbb{R}^{d}, \quad \mathfrak{R}_{i}\left(\boldsymbol{x}-\boldsymbol{x}^{\prime}\right)=\prod_{j=1}^{d} \exp \left(-\frac{\pi}{4}\left(\frac{x_{j}-x_{j}^{\prime}}{\ell_{c}^{i j}}\right)^{2}\right),
$$

for all $1 \leqslant i \leqslant n, \ell_{c}^{i j}$ being interpreted as the correlation length of the Gaussian random field $\left\{\Xi_{i}(\boldsymbol{x}), \boldsymbol{x} \in \Omega\right\}$ along the direction defined by the unit vector $\boldsymbol{e}^{j}$. The choice of such a Matérn-type (squared exponential) correlation function follows from its wide use in applications (especially in geophysics; see [1] for instance), as well as from the properties (e.g. infinite differentiability) inherited by the associated Gaussian field. Here, the generation of the stationary $\mathbb{R}$-valued Gaussian random fields is performed by using the circulant embedding method introduced in [6] and [50] (see also [5]; see [21] [49] for extensions). For illustration purposes, the random field is sampled over a regular grid with 8 points along each direction and the simulation is performed with the following parameters: $\ell_{c}^{i j}=2$ for $1 \leqslant i \leqslant 3$ and $1 \leqslant j \leqslant 2$; $f=10 ; \tau=10^{6} ; \gamma^{0}=2^{-8}$. A convergence analysis (similar to those performed in the preceding sections; see e.g. $\S 5.2 .2$ ) shows that an independent realization is obtained after 60,000 iterations. The CPU time required to generate one realization of the 
random field is 15.98 seconds on a $2.8 \mathrm{GHz}$ single core processor - note that this CPU time mainly depends on the algorithms that are used for simulating a $\mathbb{R}$-valued centered homogeneous Gaussian random field (which has to be generated at least $n \times r^{*}$ times; see e.g. [21] and the references therein for a discussion about computational complexity). Since numerical evidences show that the algebraic form of the input correlation functions is indeed preserved through the nonlinear transformation [12], the estimated correlation function (along a given direction) can be fitted to a squared exponential one by a least-square algorithm, hence providing estimates for the correlation lengths associated with the generated fields. The comparison between the estimated correlation function and its least-square fit in the direction specified by $\boldsymbol{e}^{1}$ is shown in Fig. 5.14 (left panel) for the random field $\left\{U_{1}(\boldsymbol{x}), \boldsymbol{x} \in \Omega\right\}$ (the statistical estimator being computed from a set of 200 realizations). The graphs of the fitted correlation functions (along the same direction) for the three generated random fields $\left\{U_{1}(\boldsymbol{x}), \boldsymbol{x} \in \Omega\right\},\left\{U_{2}(\boldsymbol{x}), \boldsymbol{x} \in \Omega\right\}$ and $\left\{U_{3}(\boldsymbol{x}), \boldsymbol{x} \in \Omega\right\}$ can be found in Fig. 5.14 (right panel) as well. The correlation length along the considered direction for the random
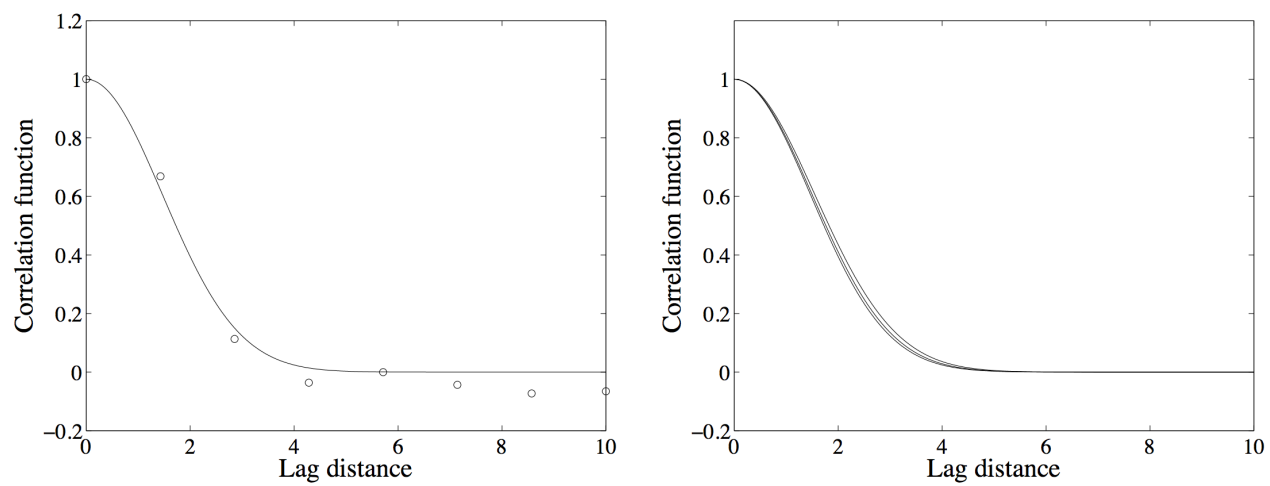

Fig. 5.14. Plot of the correlation function along the direction defined by $\boldsymbol{e}^{1}$. Left panel: correlation function associated with $\left\{U_{1}(\boldsymbol{x}), \boldsymbol{x} \in \Omega\right\}$ (circle marker: estimated values; solid line: leastsquare fit). Right panel: fitted correlation functions associated with $\left\{U_{1}(\boldsymbol{x}), \boldsymbol{x} \in \Omega\right\},\left\{U_{2}(\boldsymbol{x}), \boldsymbol{x} \in \Omega\right\}$ and $\left\{U_{3}(\boldsymbol{x}), \boldsymbol{x} \in \Omega\right\}$.

field $\left\{U_{1}(\boldsymbol{x}), \boldsymbol{x} \in \Omega\right\}$ (resp. $\left\{U_{2}(\boldsymbol{x}), \boldsymbol{x} \in \Omega\right\}$ and $\left\{U_{3}(\boldsymbol{x}), \boldsymbol{x} \in \Omega\right\}$ ) is found to be 1.84 (resp. 1.88 and 1.94).

6. Conclusion. In this work, we addressed the construction of a generator for a large class of non-Gaussian random fields with values in some subset of $\mathbb{R}^{n}$. The numerical strategy involves two main ingredients. The first one is the definition, through a regularization procedure, of a sequence of auxiliary random fields converging towards the target random field. The second ingredient is the derivation of a discretization algorithm which is defined by combining an extension of an adaptivity procedure recently proposed in the literature with an explicit Verlet scheme. The scheme thus obtained allows refining the integration step on-the-fly, hence preventing the scheme from diverging as the current state goes closer to the boundary of the admissible set. The proposed strategy was finally exemplified on three examples that illustrate both the adaptivity procedure and the convergence of the algorithm.

Acknowledgments. This research was funded by the French National Research Agency (ANR) under TYCHE (ANR-2010-BLAN-0904) and MOSAIC (ANR-12-JS090001-01) contracts. 


\section{REFERENCES}

[1] T. V. Apanasovich, M. G. Genton and Y. Sun , A Valid Matérn Class of Cross-Covariance Functions for Multivariate Random Fields With Any Number of Components, Journal of the American Statistical Association, 107 (2012), pp. 180-193.

[2] M. Arnst, R. G. Ghanem And C. Solze, Identification of Bayesian posteriors for coefficients of chaos expansions, Journal of Computational Physics, 229(9) (2010), pp. 3134-3154.

[3] P. Bocchini and G. Deodatis, Critical review and latest developments of a class of simulation algorithms for strongly non-Gaussian random fields, Probabilistic Engineering Mechanics, 23(4) (2008), pp. 393-407.

[4] Z. I. Botev, J. F. Grotowski and D. P. Kroese, Kernel density estimation via diffusion, Annals of Statistics, 38 (2010), pp. 2916-2957.

[5] G. Chan And A. T. A. Wood, Simulation of stationary Gaussian vector fields, Statistics and Computing, 9 (1999), pp. 265-268.

[6] C. Dietrich and G. Newsam, A Fast and Exact Simulation of Stationary Gaussian Processes Through Circulant Embedding of the Covariance Matrix, SIAM Journal of Scientific Computing, 18(4) (1997), pp. 1088-1107.

[7] P. E. Kleden and E. Platen, Numerical Solution of Stochastic Differential Equations, Springer, Berlin, 1992.

[8] P. Kree and C. Solze, Mathematics of Random Phenomena, Reidel Publishing Company, Dordrecht, Holland, 1986.

[9] J. Guilleminot and C. Soize, Non-Gaussian positive-definite matrix-valued random fields with constrained eigenvalues: application to random elasticity tensors with uncertain material symmetries, International Journal for Numerical Methods in Engineering, 88(11) (2011), pp. 1128-1151.

[10] J. Guilleminot, A. Noshadravan, R. G. Ghanem and C. Soize, A probabilistic model for bounded elasticity tensor random fields with application to polycrystalline microstructures, Computer Methods in Applied Mechanics and Engineering, 200(17-20) (2011), pp. 16371648.

[11] J. Guilleminot and C. Soize, Generalized Stochastic Approach for Constitutive Equation in Linear Elasticity: A Random Matrix Model, International Journal for Numerical Methods in Engineering, 90(5) (2012), pp. 613-635.

[12] J. Guilleminot and C. Solze, Prior modeling and generator for non-Gaussian tensor-valued random fields with symmetry properties. Application to elastic random media, SIAM Multiscale Modeling \& Simulation, 11(3) (2013), pp. 840-870.

[13] J. Guilleminot And C. Soize, On the statistical dependence for the components of random elasticity tensors exhibiting material symmetry properties, Journal of Elasticity, 111 (2013), pp. 109-130.

[14] E. HAirer, C. Lubich And G. WAnner, Geometric numerical integration illustrated by the Störmer/Verlet method, Acta Numerica, 12 (2003), pp. 399-450.

[15] E. HAIRER AND G. SöRdERLIND, Explicit, time reversible, adaptive step size control, SIAM J. Sci. Comput., 26(6) (2005), pp. 1838-1851.

[16] E. Hairer, C. Lubich and G. Wanner, Geometric Numerical Integration. StructurePreserving Algorithms for Ordinary Differential Equations, Springer, Berlin, 2002.

[17] R. De Vogelaere, Methods of integration which preserve the contact transformation property of the Hamiltonian equations, Report No. 4, University of Notre Dame, 1956.

[18] R. V. Field AND M. GRIGORIU, A method for the efficient construction and sampling of vectorvalued translation random fields, Probabilistic Engineering Mechanics, 29 (2012), pp. 79-91.

[19] M. Frigo And S. G. Johnson, The Design and Implementation of FFTW3, Proceedings of the IEEE, Special issue on "Program Generation, Optimization, and Platform Adaptation", 93(2) (2005), pp. 216-231.

[20] R. Ghanem and P. D. Spanos, Stochastic finite elements: A spectral approach, SpringerVerlag, New-York, 1991.

[21] T. Gneiting, H. Seveikova, D. B. Percival, M. Schlather and Y. Jiang, Fast and exact simulation of large Gaussian lattice systems in $\mathbb{R}^{2}$ : Exploring the limits, Journal of Computational and Graphical Statistics, 15(3) (2006), pp. 483-501.

[22] M. GRIGORIU, Existence and construction of translation models for stationary non-Gaussian processes, Probabilistic Engineering Mechanics, 24(4) (2009), pp. 545-551.

[23] E. T. JAYNeS, Information Theory and statistical mechanics, Physical Review, 106(4) (1957), pp. $620-630$.

[24] E. T. JAYnes, Information Theory and statistical mechanics. II, Physical Review, 108(2) (1957), pp. 171-190. 
[25] W. K. Hastings, Monte Carlo sampling methods using Markov chains and their applications, Biometrika, 109 (1970), pp. 57-97.

[26] N. Hofmann, T. Müller-Gronbach and K. Ritter, Optimal approximation of stochastic differential equations by adaptive step-size control, Mathematics of Computation, 69(231) (2000), pp. 1017-1034.

[27] N. Hofmann, T. Müller-Gronbach and K. Ritter, The Optimal Discretization of Stochastic Differential Equations, Journal of Complexity, 17 (2001), pp. 117-153.

[28] H. Lamba, J. C. Mattingly and A. M. Stuart, An adaptive Euler-Maruyama scheme for SDEs: convergence and stability, IMA Journal of Numerical Analysis, 27 (2007), pp. 479506.

[29] D. Lamberton and G. Pagès, Computation of the Invariant Distribution of a Diffusion, Bernoulli, 8(3) (2002), pp. 367-405.

[30] B. Leimkuhler AND C. Matthews, Rational construction of stochastic numerical methods for molecular sampling, Appl. Math. Res. Express, 1 (2013), pp. 34-56.

[31] V. Lemaire, An adaptive scheme for the approximation of dissipative systems, Stochastic Processes and their Applications, 117 (2007), pp. 1491-1518.

[32] O. P. Le Maître and O. M. KNiO, Spectral Methods for Uncertainty Quantification: With Applications to Computational Fluid Dynamics, Springer, New-York, 2010.

[33] M. Matsumoto And T. Nishimura, Mersenne Twister: A 623-dimensionally equidistributed uniform pseudorandom number generator, ACM Trans. on Modeling and Computer Simulation, 8(1) (1998), pp., January pp. 3-30.

[34] J. C. Mattingly, A. M. Stuart and D. J. Higham, Ergodicity for SDEs and approximations : locally Lipschitz vector fields and degenerate noise, Stochastic Process. Appl., 101(2) (2002), pp. 185-232.

[35] N. Metropolis, A. W. Rosenbluth, M. N. Rosenbluth, A. H. Teller and E. Teller, Equation of state calculations by fast computing machines, J. Chem. Phys., 21 (1953), pp. 1087-1092.

[36] G. N. Milstein and M. V. Tretyakov, Stochastic Numerics for Mathematical Physics, Springer, 2004.

[37] G. N. Milstein and M. V. Tretyakov, Quasi-symplectic methods for Langevin-type equations, IMA J. Numer. Anal., 23(4) (2003), pp. 593-626.

[38] T. Müller-Gronbach, The optimal uniform approximation of systems of stochastic differential equations, The Annals of Applied Probability, 12(2) (2002), pp. 664-690.

[39] G. O. Roberts, A. Gelman And W.R. Gilks, Weak convergence and optimal scaling of random walk Metropolis algorithms, The Annals of Applied Probability, 7(1) (1997), pp. 110120.

[40] G. Perrin, C. Soize, D. Duhamel and C. Fünfschilling, Identification of polynomial chaos representations in high dimension from a set of realizations, SIAM Journal on Scientific Computing, 34(6) (2012), pp. 2917-2945.

[41] B. Puig, F. Poirion And C. Solze, Non-Gaussian simulation using Hermite polynomial expansion: convergences and algorithms, Probabilistic Engineering Mechanics, 15(3) (2000), pp. 277-294.

[42] M. D. Shields, G. Deodatis and P. Bocchini, A simple nd efficient methodology to approximate a general non-Gaussian stochastic process by a translation process, Probabilistic Engineering Mechanics, 26(4) (2011), pp. 511-519.

[43] C. Solze, A nonparametric model of random uncertainties for reduced matrix models in structural dynamics, Probabilistic Engineering Mechanics, 15(3) (2000), pp. 277-294.

[44] C. SoIze, Non-Gaussian positive-definite matrix-valued random fields for elliptic stochastic partial differential operators, Computer Methods in Applied Mechanics and Engineering, 195(1-3) (2006), pp. 26-64.

[45] C. Solze, Construction of probability distributions in high dimension usign the maximum entropy principle: Applications to stochastic processes, random fields and random matrices, International Journal for Numerical Methods in Engineering, 76 (2008), pp. 1583-1611.

[46] C. Soize And R. G. Ghanem, Physical systems with random uncertainties: chaos representations with arbitrary probability measure, SIAM Journal on Scientific Computing, 26 (2004), pp. 395-410.

[47] C. Solze, A computational inverse method for identification of non-Gaussian random fields using the Bayesian approach in very high dimension, Computer Methods in Applied Mechanics and Engineering, 200(45-46) (2011), pp. 3083-3099.

[48] C. Solze, The Fokker-Planck Equation for Stochastic Dynamical Systems and its Explicit Steady State Solutions, World Scientific, Singapore, 1994.

[49] M. L. Stein, Fast and exact simulation of fractional Brownian motion, Journal of Computa- 
tional and Graphical Statistics, 11(3) (2002), pp. 587-599.

[50] A. T. A. Wood, G. Chan, Simulation of Stationary Gaussian Processes in $[0 ; 1]^{d}$, Journal of Computational and Graphical Statistics, 3 (1994), pp. 409-432. 